\title{
Mental compression of binary sequences in a language of thought
}

Samuel Planton ${ }^{1}$, Timo van Kerkoerle ${ }^{1}$, Leïla Abbih ${ }^{1}$, Maxime Maheu $^{1,2}$, Florent Meyniel ${ }^{1}$, Mariano Sigman ${ }^{3,4,5}$, Liping Wang ${ }^{6}$, Santiago Figueira ${ }^{4,7}$, Sergio Romano ${ }^{4,7}$, Stanislas Dehaene $^{1,8}$

${ }^{1}$ Cognitive Neuroimaging Unit, CEA, INSERM, Université Paris-Sud, Université Paris-Saclay, NeuroSpin center, 91191 Gif/Yvette, France

${ }^{2}$ Université de Paris, 75006 Paris, France

${ }^{3}$ Laboratorio de Neurociencia, Universidad Torcuato Di Tella, Buenos Aires, Argentina

${ }^{4}$ CONICET (Consejo Nacional de Investigaciones Científicas y Tecnicas), Argentina

${ }^{5}$ Facultad de Lenguas y Educacion, Universidad Nebrija, Madrid, Spain

${ }^{6}$ Institute of Neuroscience, Key Laboratory of Primate Neurobiology, CAS Center for Excellence in Brain Science and Intelligence Technology, Chinese Academy of Sciences, Shanghai, 200031, China

${ }^{7}$ Universidad de Buenos Aires. Facultad de Ciencias Exactas y Naturales. Departamento de Computacion, Buenos Aires, Argentina

${ }^{8}$ Collège de France, 11 Place Marcelin Berthelot, 75005 Paris, France

\section{Author note}

This research was supported by Inserm, CEA, Collège de France, the Bettencourt-Schueller Foundation and an ERC grant "NeuroSyntax" to S.D.,

We have no known conflict of interest to disclose.

Correspondence concerning this article should be addressed to Samuel Planton, Cognitive Neuroimaging Unit, CEA, INSERM, Université Paris-Sud, Université Paris-Saclay, NeuroSpin center, 91191 Gif/Yvette, France Contact: $\underline{\text { samuel.planton@ @ea.fr }}$ 


\begin{abstract}
The capacity to store information in working memory strongly depends upon the ability to recode the information in a compressed form. Here, we tested the theory that human adults encode binary sequences of stimuli in memory using a recursive compression algorithm akin to a "language of thought", and capable of capturing nested patterns of repetitions and alternations. In five experiments, we probed memory for auditory or visual sequences using both subjective and objective measures. We used a sequence violation paradigm in which participants detected occasional violations in an otherwise fixed sequence. Both subjective ratings of complexity and objective sequence violation detection rates were well predicted by complexity, as measured by minimal description length (also known as Kolmogorov complexity) in the binary version of the "language of geometry", a formal language previously found to account for the human encoding of complex spatial sequences in the proposed language. We contrasted the language model with a model based solely on surprise given the stimulus transition probabilities. While both models accounted for variance in the data, the language model dominated over the transition probability model for long sequences (with a number of elements far exceeding the limits of working memory). We use model comparison to show that the minimal description length in a recursive language provides a better fit than a variety of previous encoding models for sequences. The data support the hypothesis that, beyond the extraction of statistical knowledge, human sequence coding relies on an internal compression using language-like nested structures.
\end{abstract}

\title{
Keywords
}

Sequence processing; language of thought; complexity; novelty detection; statistical learning 
Sequence processing, the ability to encode and represent in memory a temporally ordered series of discrete elements, plays a central role in numerous human activities, including language. In the 1950’s, Karl Lashley (1951) and Noam Chomsky (Chomsky, 1957) famously argued, against the thendominant Skinnerian view, that sentences could not be viewed as mere temporal sequences of successive words, but rather were mentally represented as tree structures, with potentially unlimited embeddings of phrases within phrases. Here, we tested whether a similar postulation of nested structures is required in order to account for a much simpler feat than language processing: the human memorization of simple binary sequences, i.e. sequences informed by two elements A and B (e.g. high and low pitch, or red and green dots).

Understanding how humans and other animals encode and represent temporal sequences has recently emerged as a crucial issue in the study of comparative cognition, as it allows a direct comparison between species (Dehaene, Meyniel, Wacongne, Wang, \& Pallier, 2015; Wilson, Marslen-Wilson, \& Petkov, 2017). Recursive phrase structures have been proposed to lie at the core of the human language faculty (Hauser, Chomsky, \& Fitch, 2002), this hypothesis was later extended to the idea that a competence for nested trees underlies other uniquely human cognitive abilities such as mathematics or music (Conway \& Christiansen, 2001; Dehaene et al., 2015; Fitch, 2014; Hauser \& Watumull, 2017). More specifically, several abstract formats to encode sequences are available to non-human animals: a recent review (Dehaene et al., 2015) distinguished four levels including statistical regularities such as transition probabilities, ordinal regularities (what comes first, second, etc.), recurring chunks, and algebraic patterns (Fujii, 2003; Jiang et al., 2018; Marcus, Vijayan, Bandi Rao, \& Vishton, 1999; Wang, Uhrig, Jarraya, \& Dehaene, 2015; Wilson et al., 2013). In contrast, only humans are suggested to possess a representation of nested trees (Dehaene et al., 2015), also being described as a "universal generative faculty" (Hauser \& Watumull, 2017) or "language of thought" (Amalric et al., 2017; Fodor, 1975) capable of encoding arbitrarily nested rules.

Here we tested the hypothesis that humans spontaneously encode sequences using nested tree structures. To be able to do this, three steps are needed. First, one must generate systematic data on human subjects' encoding of sequences. Second, one must determine the specific mental operations that 
characterize the language used by humans to encode sequence structures in memory. Third, that language must be shown to provide a tight fit to the data, superior to simpler predictors such as statistical learning. Such a validation is a crucial and necessary step before attempting to understand the alleged specificity of the language model for humans relative to other species.

We argue here that this goal can be achieved by using the simplest form of temporal sequences, namely binary sequences. Indeed, as opposed to more complex sequences, such as the ones of the natural language, which involve numerous factors difficult to properly control (prior knowledge, semantic content, word frequency, etc.), binary sequences allow to easily control the sensory content of the input, and are potentially accessible to a wide variety of populations beyond human adults. They nevertheless preserve the possibility of forming structures at different hierarchical levels, from statistical relationships to language-like rules, and thus of arbitrating between different models of sequence encoding.

\section{A short review of sequence complexity}

The concept of compression in working memory is an essential ingredient of the present work. Much prior research shows that human memory is not simply determined by the number of words, digits or locations that must be remembered, but also by their capacity to be "compressed" into a smaller number of known phrases, groups, or chunks (Brady, Konkle, \& Alvarez, 2009; Chase \& Ericsson, 1982; Cowan, 2001; Ericsson, Chase, \& Faloon, 1980; J. Feldman, 2000; Gilchrist, Cowan, \& NavehBenjamin, 2008; Miller, 1956). Rather than encoding the complete series, individuals are thought to spontaneously reorganize the items so as to improve their storing in working memory. Indeed, half a century of behavioral studies have shown that accuracy in sequence encoding and production tasks varies according to the complexity of the sequence. Glanzer and Clark (1963) already proposed to use the length of the most compact internal description of a sequence as a measure of its complexity. They indeed found that the number of words that participants used to describe an array of eight symbols, each colored either in black or in white, was correlated with the accuracy in reproducing it. Such mean 
verbalization length (MVL) predicted behavior better than a simple count of the number of runs in the sequence (e.g., "AAABBBAA" has three runs), particularly for the "ABABABAB", which could be simply described as "alternating".

Generalizing upon this early work, one may propose that the complexity of a sequence relates to the length of its compressed form when it is recoded using an internal language. Consistent with such idea, Restle and Brown (1970) showed that participants learned a series of 10 button presses, not as an associative chain of elements, but by encoding it as an abstract pattern, defined as the set of rules that were needed to generate it. The profile of errors suggested that participants represented the sequences as hierarchical trees of embedded rules (i.e., repetition, transposition, mirroring), equivalent to the tree structures found in language (Restle, 1970). The psychological reality of this proposal was strengthened by showing that performance decreased precisely at the boundaries of higher hierarchical level groups of elements (Restle, 1970, 1973; Restle \& Brown, 1970). However, this approach was not developed into a full-blown universal language explaining how any sequence or pattern would be encoded.

A more formal approach for estimating the complexity of patterns, usually referred to as algorithmic complexity, program size complexity or Kolmogorov complexity (KC), was proposed by Kolmogorov (1965) and Chaitin (1969), within the framework of Algorithmic Information Theory. They defined the complexity of a sequence as the length of the shortest computer program capable of producing it. $\mathrm{KC}$ therefore corresponds to the minimal description length (MDL) with respect to a Turing-complete language, which means that it is capable of encoding any computable function. However, this theory had several practical difficulties to be used in psychological research. First, it was conceived for infinite sequences. Second, for any turing-complete language, $\mathrm{KC}$ is not computable, in the sense that no computer program can determine as output the value of the complexity of any input sequence. However, $\mathrm{KC}$ can be approximated in a computable way, and one way to do it is by considering domain-specific languages that encode a finite set of relevant function (i.e. a semantics) (Romano, Sigman, \& Figueira, 2013). Recently, the group of Gauvrit, Delahaye, Zenil and SolerToscano proposed an approximation to $\mathrm{KC}$ using the coding theorem as starting point; this theorem relates the algorithmic complexity of a sequence to the probability that a universal machine outputs such 
sequence (Delahaye \& Zenil, 2012; Gauvrit, Singmann, Soler-Toscano, \& Zenil, 2016; Gauvrit, Zenil, Delahaye, \& Soler-Toscano, 2014; Soler-Toscano, Zenil, Delahaye, \& Gauvrit, 2014). They provided algorithmic complexity measures for a large set of short sequences. This proposal was presented as the best approximation of "an ultimate measure of randomness" and appeared to predict the biases observed when individuals are asked to either judge the randomness of patterns or to produce random patterns (Gauvrit et al., 2016, 2014).

As an alternative to algorithmic complexity, Aksentijevic and Gibson (2012) proposed another measure of sequence complexity, based on the notion of "change" (the inverse of invariance), which they called change complexity. They argued that humans attend to the structural information conveyed by the transition from one element to the next, rather than the symbols themselves. Change complexity is thus computed by quantifying the average amount of change across all sub-sequences contained in a sequence. Aksentijevic and Gibson (2012) further show that their measure has interesting properties such as a sensitivity to periodicity and symmetries, and that it performs better than previously proposed measures in predicting objective behavioral performance and subjective complexity of sequences.

As stated above, a proposal tightly related to $\mathrm{KC}$ is that human subjects compress sequences internally, not necessarily using a set of instructions of a Turing-complete language, but using a variety of computer-like primitives such as for-loops, while-loops, and other routines forming a specific internal "language of though" (Fodor, 1975) strong enough to describe any sequence (or the object under study), but weak enough as to being able to compute the Kolmogorov complexity induced by this language. Such a language would allow the combination of simple primitives into complex embedded patterns or recursive rules. Language of thought models have been used with success to study different aspects of human learning (J. Feldman, 2000; Goodman, Ullman, \& Tenenbaum, 2011; Piantadosi \& Jacobs, 2016; Piantadosi, Tenenbaum, \& Goodman, 2012) and may constitute another mean to address the notion of Kolmogorov complexity. 


\section{The proposed language for binary sequence}

Here, we explore this possibility by pursuing a language of thought called the language of geometry which was previously introduced by our team to model human performance in the domain of spatial working memory (Amalric et al., 2017). In this previous work, human participants were presented with a sequence of eight locations on a regular octagon. Using both behavioral and brain-imaging data, we showed the necessity and adequacy of a computer-like language consisting of geometrical primitives of rotation and symmetry plus the ability to repeat them with various variations in starting point or symmetries (Amalric et al., 2017; Romano et al., 2018; Wang et al., 2019). This language was shown to predict which sequences appear as regular, and how educated adults, uneducated Amazon Indians and young children performed an explicit sequence completion task (Amalric et al., 2017) or simply gazed a sequence of dots as fast as possible (Wang et al., 2019). Sequence complexity, defined as minimal description length predicted behavioral performance, and could account for variability in brain activation patterns (Amalric et al., 2017; Romano et al., 2018; Wang et al., 2019).

This language of geometry enables the generation of programs that encode spatial transitions. It uses primitive instructions or rules regarding the size and the direction of the next step (e.g., $+1=$ next element clockwise; $+2=$ second element clockwise), as well as the reflection over some axes (e.g., $\mathrm{H}=$ horizontal symmetry, picking the symmetrical location along a horizontal axis). Furthermore, these elements can be repeated, for instance $+1^{\wedge} 8$ describes a full clockwise turn around the octagon. Finally, those repetitions can be arbitrarily embedded. For instance, the expression $\left[[+2]^{\wedge} 4\right]^{\wedge} 2<+1>$ first draws a square, as determined by the subexpression $\left.[+2]^{\wedge} 4\right)$, then a second one with an offset of +1 in the starting point (see Amalric et al, 2017, for a full formal description).

In the present study, we test the highly constrained hypothesis that a minimal version of this language may suffice to account for the human encoding of a completely different type of sequence, namely non-spatial binary sequences composed of only two arbitrary states A, B instead of the eight locations of the octagon. For such sequences, the language can be stripped of most of its primitives. We kept only the operations of staying (" +0 ") versus moving to the other item ("b", i.e., the alternation 
instruction - or any specific symmetry in the original language), and the operation of repetition, possibly with a variation in the starting point. The language is thus able to encode any repetition of instructions in a compressed manner. The sequence "AAAA", for instance, would be denoted [+0]^4 (i.e., same state four times), the sequence “ABAB" would be denoted $[+0]^{\wedge} 4<b>$ (i.e., alternations from the initial state four times). The language is recursive and can produce nested descriptions, for instance "AABAAB" can be described as "two repetitions of [two repetitions plus one change]" (see example Figure 1A). The code is available online at https://github.com/sromano/language-of-geometry.

Given this specific proposal for the language of thought, one can compute the length of the shortest expression describing a given sequence, and use this minimum description length (MDL) as a measure of complexity. The MDL or KC relative to this language is calculated by adding a fixed cost for each primitive instruction +0 and $b$. The additional cost for the repetition of an instruction or a set of instructions $n$ times is assumed to correspond to $\log _{10}(n)$ (rounded up), i.e. the number of digits needed to encode the number in decimal notation. The relative value of the two costs is such that even a single repetition compresses an expression: $+0^{\wedge} 2$ is assumed to be more compressed than the mere concatenation of $+0+0$ (see supporting information in Amalric et al., 2017, for details). As a result, the language favors a description of sequence based on nested repetitions of instructions, thus sharply dissociating sequence length and complexity. Consistent with the "minimal description length" principle, among the multiple expressions that can describe the same sequence, the expression with the lowest complexity is considered to correspond to the human mental representation of the sequence (and its length approximates the Kolmogorov complexity of the sequence; see Grunwald, 2004; Romano et al., 2013). In a nutshell, the assumption is that, in order to minimize memory load, participants mentally compress the sequence structure using the proposed formal language. 


\section{Probing memory for sequences: The sequence violation}

\section{paradigm}

In preparation for future experiments involving infants or non-human animals, it is useful to probe sequence processing using a paradigm that does not require language skills, nor explicit production of responses. A classic approach consists in introducing rare violations in an otherwise regular sequential input. At the most elementary level, in the oddball paradigm, the simple repetition of an auditory or visual stimulus with a regular timing suffices for the brain to generate expectations, such that the unexpected violation of this regularity, by suddenly replacing the stimulus by a different one, gives rise to an automatic surprise or novelty response. Such a surprise effect can be detected behaviorally, e.g. using an explicit detection, a pupillary response, or electrophysiological signatures including the mismatch negativity (Garrido, Kilner, Stephan, \& Friston, 2009; Näätänen, 2003; Squires, Squires, \& Hillyard, 1975).

A more complex brain response to novelty arises in the local/global paradigm (Bekinschtein et al., 2009; Wacongne et al., 2011), which contrasts two levels of violation: a local one, when a B stimulus follows a series of As (as in "AAAAB"); and a global one where, at a higher hierarchical level, the habitual sequence (e.g. "AAAAB" repeated multiple times) is replaced by a difference sequence (e.g. "AAAAA"). The use of this paradigm with neuroimaging made it for instance possible to show that macaques brains tend to spontaneously encode simple sequential patterns, using a cerebral network similar to the one in humans (Chao, Takaura, Wang, Fujii, \& Dehaene, 2018; Uhrig, Dehaene, \& Jarraya, 2014; Wang et al., 2015), or that such ability is already present in human infants (Basirat, Dehaene, \& Dehaene-Lambertz, 2014). It was also successfully used to show, with asleep participants or unconscious patients, that the processing of auditory sequential inputs at the global level (i.e., the level of patterns) is mainly restricted to conscious processing (Bekinschtein et al., 2009; Faugeras et al., 2011; Strauss et al., 2015). Behavioral and hemodynamic novelty responses to violations were also used by Huettel et al. (2002) to show that human adults spontaneously encoded simple repeating and alternating 
patterns, and that their response times and fMRI frontal activity patterns varied when such a local pattern was violated. Interestingly, the strength of the novelty response observed when the pattern was broken, was proportional to the length of the preceding pattern, suggesting that the novelty response could perhaps track sequence complexity.

Here, we test the hypothesis that the violation detection task can be used to probe the encoding of sequences of higher level of complexity, thus revealing their degree of psychological regularity and give an insight into the internal language of thought used to encode them. While we focus on explicit behavioral responses in a violation detection task, we do this with the aim of paving the way to future studies using non-verbal subjects or using brain measures of implicit violation detection.

\section{Statistical learning in sequence processing}

A language of thought is by no means the only way to encode binary sequences. At a lower level of abstraction, the detection of sequential structures in the environment may involve the identification of statistical regularities in the frequencies of events or the transitions between them (Dehaene et al., 2015; Maheu, Dehaene, \& Meyniel, 2019). Even in the language domain, transition probabilities are known to play an important role. Eight-month-old infants have for instance been shown to rely on transition probabilities between syllables in order to segment a continuous stream of syllables into distinct words (Romberg \& Saffran, 2010; Saffran, Aslin, \& Newport, 1996). Transition probability learning, revealed by the observation of a novelty response to an improbable event, was also reported in the visual modality (Abla \& Okanoya, 2009; Kirkham, Slemmer, \& Johnson, 2002), as well as in nonhuman primates (Hauser, Newport, \& Aslin, 2001; Meyer \& Olson, 2011). This process appears to be automatic and continues to operate under non-conscious conditions (Bekinschtein et al., 2009; Faugeras et al., 2011; Strauss et al., 2015). When using the novelty effect as an indicator of sequence complexity, it is therefore essential to separate the respective contributions of statistical learning and of a putative language of thought. 
Computational models relying on probabilistic inference have been proposed for statistical learning. Mars et al., (2008) for instance showed that the trial-by-trial modulation of the amplitude of the P300 (an event-related potential response associated with unexpected events) could be explained by a model tracking the frequency of occurrence of items (among 4) in a temporal sequence. Similarly, our team proposed a Bayesian model for the acquisition of transition probabilities (not simply item frequency), and showed that it could explain a great variety of different behavioral and brain observations in binary sequence processing experiments (Maheu et al., 2019; Meyniel, Maheu, \& Dehaene, 2016). The degree of confidence in a prediction can furthermore be predicted using such computational approach (Meyniel \& Dehaene, 2017). In these models, Shannon surprise, a mathematical measure of the improbability of an event considering past events (the negative log probability of events) (Friston, 2010; Shannon, 1948; Strange, Duggins, Penny, Dolan, \& Friston, 2005), is considered a good predictor of behavioral and neural responses.

It is likely that two distinct systems for statistic versus rule-based learning co-exist in the human brain. What is unknown is whether they operate independently and whether one is privileged at the expense of the other depending on the nature of the information to be encoded. We argue that any attempt to uncover the specific cognitive mechanisms behind rule learning in humans, especially in comparison with other species, must take into account the contribution of the less abstract yet powerful prediction system based on the statistical properties of events.

\section{The current study}

In summary, we propose to test the hypothesis that, when confronted with a sequential input, individuals tend to spontaneously recode the sequence in an abstract form, using an internal "language of thought" composed of a limited set of simple rules that can be hierarchically embedded. We compare this language of thought hypothesis to other computational approaches to sequence complexity as well as to the transition-probability model. 
We therefore conducted a series of behavioral experiments in which participants were asked to listen to short auditory binary sequences (alternations of a sound "A" and a sound "B"), whose statistical properties and predicted complexity varied. Learning was assessed by examining the capacity of participants to detect rare violations of the learned sequence (i.e. when one tone was replaced by the other). Our hypothesis was that, for equal sequence length, error rate and response time in violation detection would increase in parallel with sequence complexity. In some experiments, in addition to those objective indices of complexity, we also asked participants to report subjective rating of complexity. Finally, in one experiment, we also compared auditory and visual sequences to test whether our findings were dependent on the sensory modality.

For analysis, we examine if the results correlated with the minimal description length in the proposed language of thought (hereafter called LoT-complexity to distinguish it from other complexity measures). To separate rule-based and statistical learning, we compared LoT-complexity and surprise as predictors of performance. We started with long sequences of 16 items, and then probed the adequacy of the proposed language to shorter sequences. A simple prediction is that shorter sequences, are more likely to be stored in a verbatim representation in working memory, without any internal compression. Thus, we predicted that the effect of MDL in the proposed language of thought would decrease as the sequence gets shorter. On the other hand, given the automaticity of statistical learning, we did not expect any difference in its contribution to long versus short sequences.

\section{Experiment 1 (16-items long auditory sequences)}

In experiment 1 , we selected 10 auditory sequences of 16 items, a number that vastly exceeds working memory capacity, which is typically between 4 to 9 items (Cowan, 2001, 2010; Miller, 1956). All sequences had equal numbers of sounds $\mathrm{A}$ and B (to reduce confounds related to the relative probability of As and Bs), yet they varied widely in LoT-complexity (see Figure 2). We obtained a subjective measure of complexity as well as objective measures of complexity based on the response to occasional violations. Two types of violations were introduced: sequence deviants in which an A was replaced by a B or vice-versa; and "super-deviants", in which an A or B was replaced by a rare novel 
tone C (see Figure 1B). We predicted that the detection of sequence deviants would be affected by sequence complexity, since responding to them required the detection of a discrepancy between the observed and the predicted stimuli, and such a prediction would be more difficult for more complex sequences. Super-deviants were not expected to yield a complexity effect, however, since they deviated from other stimuli at the most basic stimulus-frequency level. Super-deviants stimuli were introduced in an effort to ensure an invariant task which would equalize level of attention in all blocks, regardless of sequence complexity.

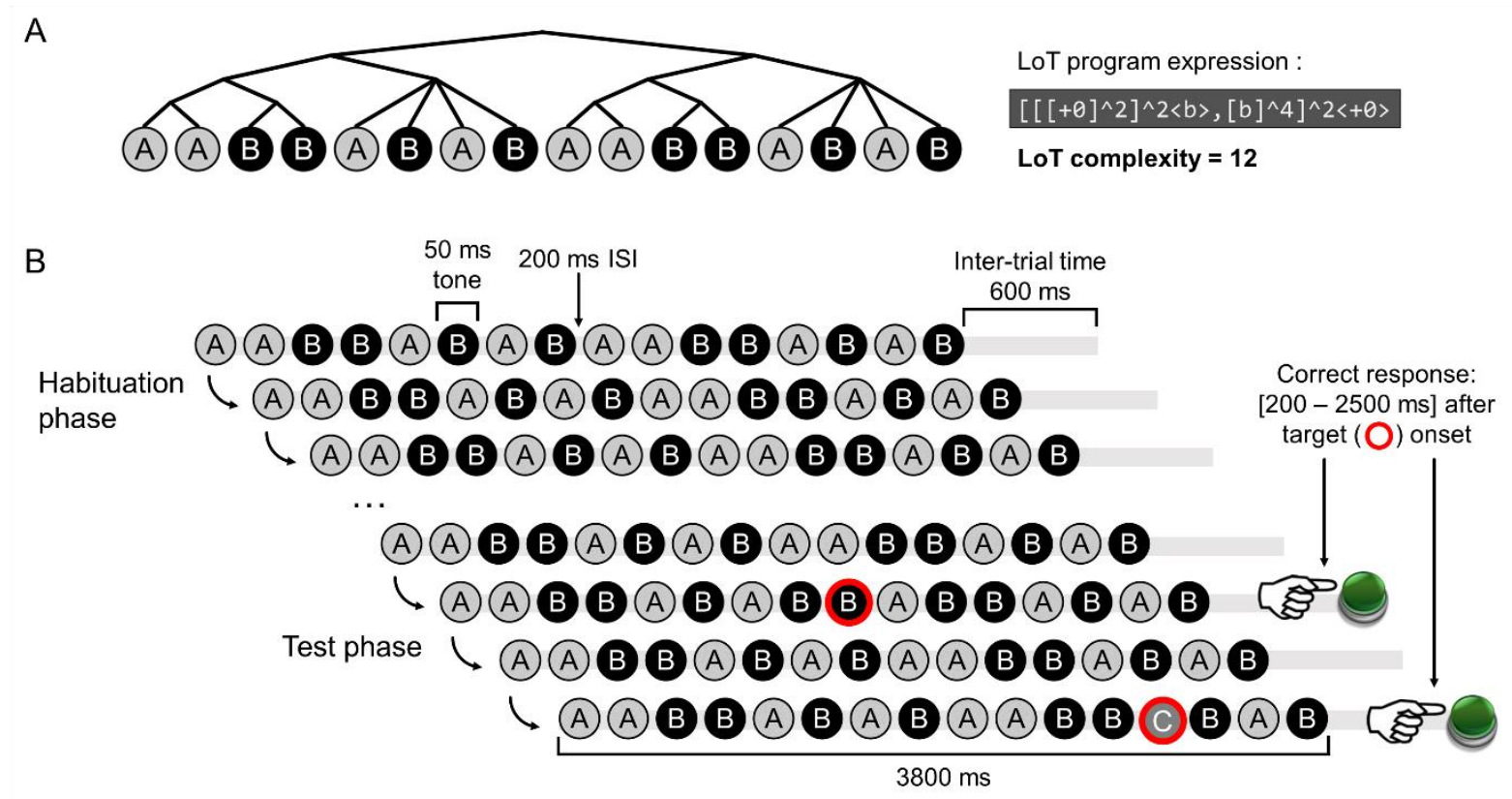

Figure 1: (A) Example of a 16-items long sequential pattern, with its shortest representation in the language of thought (i.e. LoT program expression) and the tree-structure derived from this expression (illustrating the hierarchical representation). The LoT complexity of this sequence is also indicated. (B) Experimental design of the violation detection task: a session with the sequence "AABBABABAABBABAB" is represented, with one example of a target sequence deviant item ("A" replaced by "B", at position 9) and one example of a target super-deviant item ("C" at position 13) (deviants could occur at positions 9, 11, 13 or 15$)$.

\section{Method}

\section{Participants}

Twenty-eight healthy volunteers $\left(M_{\mathrm{age}}=24.3, S D=3.2,16\right.$ women $)$ participated in the current experiment. They all gave written consent to participate and were paid for their participation. All 
participants performed the subjective complexity rating task. Due to time constraints, 7 of them performed only 6 out of the 10 independent short sessions of deviance detection.

\section{Stimuli}

Auditory binary sequences were composed of an alternation of two different tones; low pitch and high pitch. Each stimulus was a complex tone synthesized with the superimposition of four sine waves. Sound frequencies were chosen to correspond to musical notes: 494, 740, 988 and $1480 \mathrm{~Hz}$ (i.e., B, F\#, B, F\#) for the lower pitch tone, and 622, 932, 1245 and $1865 \mathrm{~Hz}$ (i.e., D\#, Bb, D\#, Bb) for the higher pitch tone. The two complex tones were randomly assigned to items A and B (i.e., the two elements composing the binary sequential patterns) for each experimental session. Thus, stimulus attribution changed from one sequence to the next and from one participant to the next but was kept constant for a given sequence in a given participant. In addition, one lower pitch tone $(415,622,831$ and $1245 \mathrm{~Hz})$ and one higher pitch tone $(740,1109,1480$ and $2217 \mathrm{~Hz})$ were synthesized, to be used as easy-to-detect super-deviant (or C) stimuli. All tones were $50 \mathrm{~ms}$ long, with $5 \mathrm{~ms}$ initial and final ramp. Inter-stimulus interval (ISI) was $200 \mathrm{~ms}$. Total sequence duration was $3800 \mathrm{~ms}$.

Ten sequences were chosen (see Figure 2), which were all composed of the same number of items ( $8 \mathrm{As}, 8 \mathrm{Bs}$ ). The first four sequences, of lowest complexity, followed the simple algebraic pattern $\left(\mathrm{A}^{n} \mathrm{~B}^{n}\right)^{x}:(\mathrm{AB})^{8},\left(\mathrm{~A}^{2} \mathrm{~B}^{2}\right)^{4},\left(\mathrm{~A}^{4} \mathrm{~B}^{4}\right)^{2}$ and $\mathrm{A}^{8} \mathrm{~B}^{8}$. The period of these sequences differed $(2,4,8$ and 16 tones $)$, but the complexity was identical (LoT complexity $=6$ ). The other 6 sequences had LoT complexity values ranging from 12 to 23 . Half of them were periodic (period of 8). 


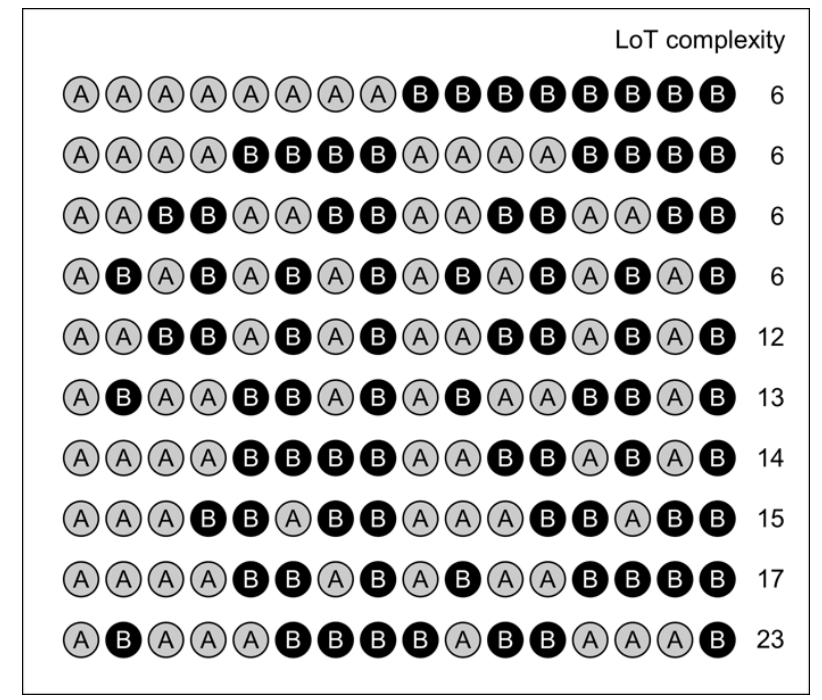

Figure 2: Ten 16-items long sequential patterns used in Experiment 1, with their corresponding LoT complexity value.

\section{Procedure}

Participants were seated in front of a computer in a quiet room and were wearing headphones. Stimuli were delivered using the Psychophysics Toolbox 3 (Brainard \& Vision, 1997; Kleiner et al., 2007) running on Matlab R2016a (Mathworks Inc., Natick, MA, USA). Before starting the experiment, participants listened to a sample of the stimuli (different sequences from the ones used in the main experiment) and the sound volume was adjusted if necessary.

In the first part of the experiment, participants performed the complexity rating task. They were asked to judge each sequence on a scale going from "1: very simple" to "9: very complex", by pressing the corresponding key on the keyboard just after sequence presentation. A response was requested at each trial. Each of the ten sequences was presented three times, in a pseudo-random order (30 trials). The low-pitch and high-pitch tone were randomly assigned to either A and B or to B and A at each presentation.

In the second part, the violation detection task, each of the ten sequences was tested in a different short session of approximately $4 \mathrm{~min}$ (Figure 1B). Order of sessions was randomized for each participant. Each session comprised three blocks separated by pauses and in which the sequence (3800 ms long) was repeatedly presented with a $600 \mathrm{~ms}$ inter-trial duration. In the first block, the habituation block, the unaltered sequence was presented eight times. Participants were asked to listen to the stimuli and try to remember the sequence. In the two following blocks, the testing blocks, participants were 
asked to respond whenever they detected that the sequence had been altered (one deviant tone), by pressing the space key of the keyboard as quickly as possible (without necessarily waiting until the end of sequence presentation). Each of the two test blocks comprised 18 sequences, 9 of them containing one deviant tone (among the sixteen tones composing the sequence). Two-thirds of the deviant sequences were produced by replacing a tone A by a tone B, or conversely ("sequence deviant" tones, 12 trials per session). The remaining third were obtained by replacing one tone by a low or high-pitch C sound ("super-deviant" tone, 6 trials per session). Deviant tones could occur at only four, equally probable, positions within the second half of the sequence (positions 9, 11, 13 or 15).

\section{Data analysis}

The responses collected in the complexity rating task, ranging from 1 to 9 , were normalized for each participant using a $z$-score transformation of the raw ratings within each participant. An average complexity rating was computed for each sequence and subject and entered into a mixed effect model with participant as random factor and LoT complexity value as a fixed effect predictor. Here and in following mixed effect analyses, similar results were obtained using classical repeated-measures ANOVAs with participants as the random factor.

For the violation detection task, a button press occurring between 200 and $2500 \mathrm{~ms}$ after deviant stimulus onset was considered a hit (i.e. correct response). An absence of response during this interval was counted as a miss. False alarms were collected and analyzed separately (using a simple linear regression analysis with the LoT complexity predictor). Note that participants were not aware of the number or frequency of targets, and could respond at any time. Thus only the number of false alarms, rather than a ratio depending on the number of trials, was relevant. The Linear Integrated SpeedAccuracy Score (LISAS) (Vandierendonck, 2017, 2018), an integrated measure of response times and error rates, was used as the main indicator of performance (results with response times and miss rates were quite convergent and are provided in supplementary materials). This score was computed for each

sequence, each deviant type in each subject, according to the following formula: $=R T_{C}+M R \times \frac{S_{R T}}{S_{M R}}$, where $R T_{C}$ refers to the average response time (of correct responses), $M R$ to the miss rate, $S_{R T}$ to 
participant's overall $R T$ standard deviation and $S_{M R}$ to the participant's overall $M R$ standard deviation. These scores were computed after removing extreme response times (2.5 standard deviations $(S D)$ above or below the median in each condition and subject, $2.0 \%$ of data). Participants with excessive average miss rate over the entire session (i.e. 2.5 SD above group median), average response time and/or average number of false alarms were excluded (three participants). All data analyzes were performed in R 3.6.0 (R Core Team, 2017).

We performed statistical analyses using a mixed model in which the dependent variable was the LISAS within each participant and each cell of the design, participants were the random factor, and LoT complexity and deviant type (sequence deviants vs. super-deviant) were fixed factors. To clarify the interactions, we also computed the same mixed effect model after restricting the data to each deviant type. All computations were performed using the lme4 (Bates, Mächler, Bolker, \& Walker, 2015) and lmerTest (Kuznetsova, Brockhoff, \& Christensen, 2017) packages. P-values for each factor were obtained using Kenward-Roger approximation for degrees of freedom (Kenward \& Roger, 1997).

Since statistical trends were also expected to play a role in how participants react to deviant stimuli, another predictor, distinct from LoT complexity, was constructed. We used Shannon surprise, defined as the negative log-probability of the likelihood of an observation (Friston, 2010; Meyniel \& Dehaene, 2017; Meyniel et al., 2016; Shannon, 1948; Strange et al., 2005), to characterize how unexpected a deviant stimulus would be for an observer that tracks transition probabilities of the original sequence; for binary sequences: $p(\mathrm{~A} \mid \mathrm{A})=1-p(\mathrm{~B} \mid \mathrm{A})$ and $p(\mathrm{~A} \mid \mathrm{B})=1-p(\mathrm{~B} \mid \mathrm{B})$. Since the sequence was considered to be learnt after the habituation phase, fixed probabilities were used (rather than evolving on a trial-by-trial basis based on a recent time window, as used for instance by Maheu et al., 2019; Meyniel et al., 2016). For instance, in the $\mathrm{A}^{8} \mathrm{~B}^{8}$ sequence, $p(\mathrm{~A} \mid \mathrm{A})$ has a probability of 0.875 in the original sequence. Thus, the corresponding surprise of getting an $\mathrm{A}$ instead of a B at the $9^{\text {th }}$ position is low $\left(-\log _{2}(0.875) \approx 0.18 \mathrm{bit}\right)$. In the same sequence, $p(\mathrm{~A} \mid \mathrm{B})=0$ (since $\mathrm{B}$ is always followed by another B), and therefore the surprise of getting an A instead of a B at, say, the $11^{\text {th }}$ position, is maximal. To avoid an infinite when computing surprise, probabilities of 0 were replaced by a small but non-zero probability of $p=0.01$, capping the maximum surprise value at around 6.64 bits. To test whether this 
would affect our conclusions, complementary analyses were also conducted while excluding deviants with such zero probability. Note that, contrary to the LoT complexity, which is identical whatever the position of the deviant, surprise varies with deviant location in the sequence (up to four different values in one given block). Analyses comparing the surprise and LoT complexity predictors were performed using the same mixed model as above, including participants as random effects. To compare pair of models, we used likelihood ratio combined with chi-square statistical tests. When more than 2 models were involved, we computed the Akaike information criterion for each model (Akaike, 1998). Note that both methods penalize for model complexity (i.e. the number of predictors included in the regression), which varies depending on whether, or not, LoT complexity was included in addition to Shannon surprise (see above). Super-deviant trials were not included in these analyses.

In addition to those mixed effect statistics, we also report the results of simple regression analyses, which provide a summary view of the Pearson correlation coefficient $r$ between LoT complexity and either subjective complexity ratings or the LISAS for each sequence, after averaging across participants (this is the $r$ value reported in the figures). Supplementary figures provide this statistic for RTs and miss rates.

\section{Results and discussion}

\section{Complexity rating task}

We observed a strong positive linear relationship between the average subjective complexity ratings and the LoT complexity $(t(278)=24.6, \mathrm{p}<.0001$; Pearson correlation coefficient on the average ratings for each sequence, $r=.94$ ) (see Figure 3A). These results indicate that participants were readily able to judge whether a pattern is "more complex" than another, and that the formal language we used to compute sequence complexity is close to how individuals form such complexity judgements.

\section{Deviant type and complexity effects in the violation detection task}

We observed a linear relationship of LoT complexity and performance in the violation detection task (using LISAS). We observed main effects of LoT complexity $(t(415.0)=18.1, \mathrm{p}<.0001)$, deviant type (994 ms for sequence deviants vs. $570 \mathrm{~ms}$ for super-deviants; $t(414.4)=18.9, \mathrm{p}<.0001)$ and their 
interaction $(t(414.5)=11.7, \mathrm{p}<.0001)$. Indeed, the slope of the complexity effect was significantly stronger, by an order of magnitude, for sequence deviants as opposed to super-deviants (respectively $+51 \mathrm{~ms}$ vs. $+5 \mathrm{~ms}$ in simple regression, $t(16)=11.7, \mathrm{p}<.0001$; see Figure $3 \mathrm{~B}$, and Figure $\mathrm{S} 1$ for the corresponding results using response times or miss rate instead of LISAS). Nevertheless, separate analyses revealed that LoT complexity was a strong predictor of performance for sequence deviants $(t(193.0)=15.5, \mathrm{p}<.0001 ; r=.98)$ and also, surprisingly, for super-deviants $(t(198.5)=4.08, \mathrm{p}<.0001 ;$ $r=.72$ ) (Figure 3B). The latter effect on LISAS was however mainly driven by response times, since the average hit-rate for super-deviants was high (96\%) and weakly modulated by LoT complexity $(t(200.7)=2.32, \mathrm{p}<.03)$.

The number of false alarms per sequence (which was 1.99 on average) also increased with sequence LoT complexity $(t(214.4)=4.20, \mathrm{p}<.0001 ; r=.74)$, suggesting here again that the LoT complexity was a good predictor of the quality of sequence encoding.

The results of this first experiment with long binary auditory sequences (16 items) thus indicate that the formal language used to describe sequences in a compressed form, based on simple (possibly embedded) rules, is highly relevant to predict (1) how "complex" an auditory sequence is judged by adult participants after having listened to it once and (2) how difficult it was to learn these sequences in order to detect alterations.

Sequence complexity was expected to have little or no impact on the detection of super-deviants, i.e. high-pitch or low-pitch tones different from the two tones composing the binary auditory sequence. Our rationale was that such "C" tones were detectable even without any prior knowledge of sequence structure. While performance in detecting super-deviants was much better than for sequence deviants, even for the simplest sequences, a clear relationship between LoT complexity and performance continued to be observed. We see at least two interpretations of this finding. First, there could be an increased attentional cost of having to detect violations in more complex sequences, thus placing subjects in a dual-task setting of having to simultaneously maintain a complex representation in memory and to respond to deviants. Alternatively, the effect could reflect the influence of a top-down prediction system which would use sequence structure to generate predictions of the incoming stimuli. Complex 
sequences would be less well predicted, and this would in turn affect the speed with which any deviant is detected. We return to this question in the General Discussion.

\section{Surprise effects}

Many prior experiments, using either or both behavior and brain-imaging measures, have shown that individuals constantly entertain predictions about future observations using probabilistic knowledge based on past observations (e.g., Maheu et al., 2019; Meyniel et al., 2016). In order to test whether task performance could be explained by transition probabilities (surprise) or also implied an encoding of sequence structure, a mixed model (with participants as a random effect) including fixed effects of both LoT complexity and surprise (averaged across the 4 possible positions of deviants in a given sequence) was compared to a null model including only the latter. The effect of surprise in the null model with surprise alone was significant $(t(193.0)=5.31, \mathrm{p}<.0001)$. However, a likelihood ratio test showed that adding LoT complexity significantly improved the goodness of fit: $\chi^{2}(1)=130.9, p<.0001$. Adding a "period" factor (i.e., period values were $2,4,8$ or 16) did not improve the model fit $\left(\chi^{2}(1)=1.23, p=\right.$ .27 ), confirming the prediction that the four included $\mathrm{A}^{n} \mathrm{~B}^{n}$ patterns have the same psychological complexity, and suggesting that this information is already captured by LoT complexity. Adding the interaction between surprise and LoT complexity did not improve goodness of fit either $\left(\chi^{2}(1)=2.50, p\right.$ $=.11)$. As reported in Table 1, the LoT complexity fixed effect was significant in the final full model $(t(192.4)=13.6, \mathrm{p}<.0001)$, but not the surprise fixed effect $(t(191.8)=0.60, \mathrm{p}=.55)$. The absence of a significant effect of surprise once sequence complexity is taken into account reflects the existence of a small correlation between the two measures $(r=-.54)$, biased transition probabilities in less complex sequences tending to make deviants more easily surprising. It also shows that when this two slightly colinear factors are included, LoT is more effective than surprise describing the variance of the data.

As our choice of attributing an arbitrary padding value (0.01) to deviant transitions events with zero probability when computing surprise may have biased the results, we recomputed the LISAS and average surprise while excluding all such trials (i.e. all deviant positions in the (AB) ${ }^{8}$ pattern, 3 out of 4 deviant positions in the $\mathrm{A}^{8} \mathrm{~B}^{8}$ pattern). Here again, a likelihood ratio test showed that the goodness of fit increased significantly when adding LoT complexity to a null model containing only surprise $\left(\chi^{2}(1)=\right.$ 
$116.3, \mathrm{p}<.0001)$. However, both complexity $(t(165.5)=12.9, \mathrm{p}<.0001)$ and surprise $(t(165.8)=3.82$, $\mathrm{p}<.0001)$ were significant with this subset of the data.

In conclusion, the strong complexity effects observed here indicated that participants used some form of compression of information to encode the sequence and perform the task over and above statistical information. Although no instruction was given in that sense, this strategy may be needed in order to deal with a difficult, memory-demanding task. Indeed, at the maximum level of complexity used, performance in violation detection was very low (the violation detection rate dropped to $41 \%$ for sequence deviants). In the subsequent experiments, we asked whether similar complexity effects emerged using the same paradigm with shorter sequences; when the sequence can be more easily encoded and stored "as a whole", without necessarily requiring a re-encoding in a more abstract, compressed form. In these less demanding conditions, it can be expected that the spontaneous encoding of transitions probabilities between items will play a more important role in the detection of violations. 
A Complexity rating task

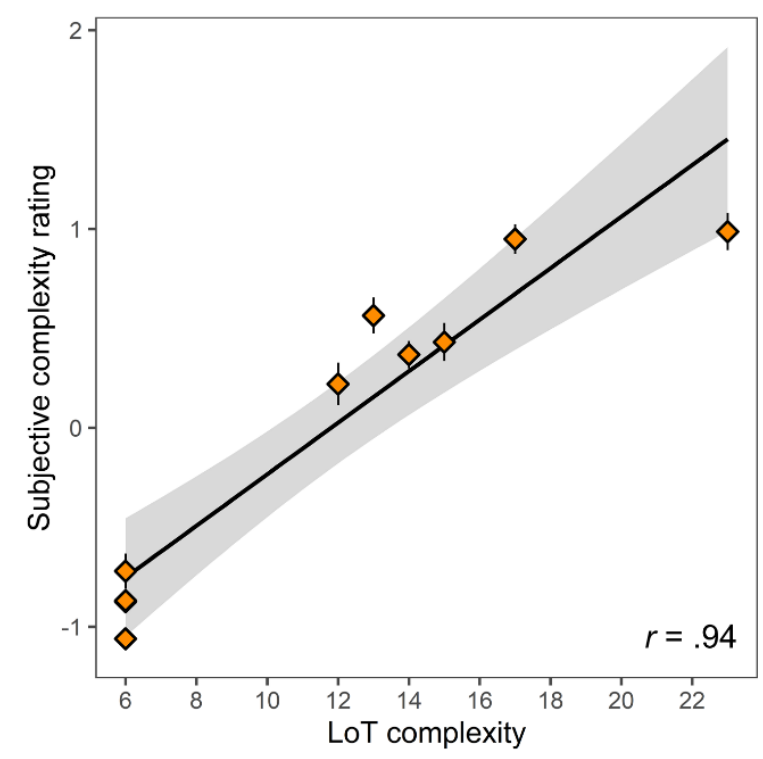

B Deviant detection task

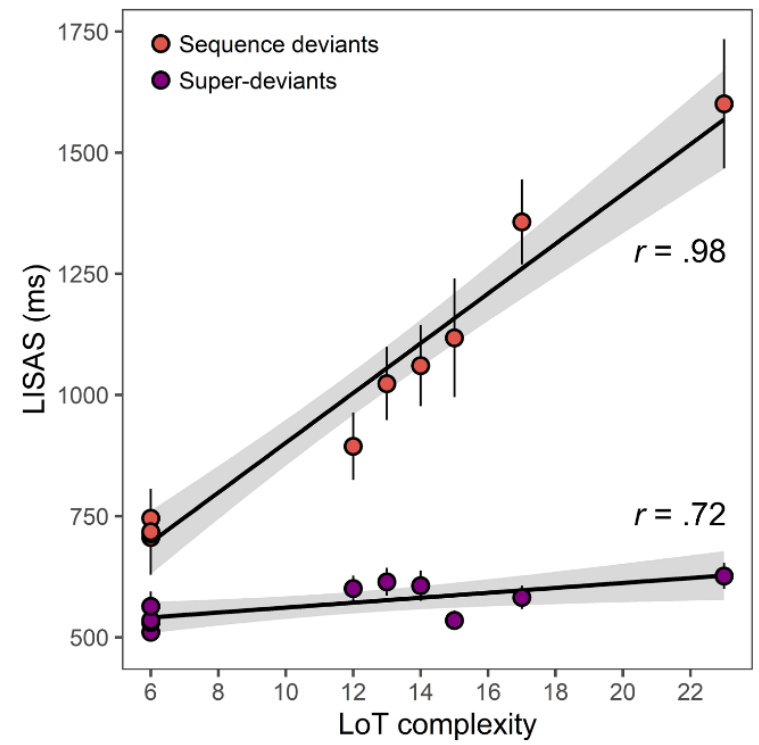

Figure 3: Linear relationship between LoT complexity and subjective and objective measures obtained in experiment 1 with ten 16-items long auditory sequences (with $95 \%$ confidence intervals bands in gray). The Pearson correlation ( $r$ ) coefficient is indicated. Each marker represents the group-average for a given sequence. Error bars represent SEM across participants. (A) LoT complexity vs. subjective complexity ratings. (B) LoT complexity vs. performance in the violation detection task (Linear Integrated Speed-Accuracy Score), for sequence deviants and super-deviants. 
Table 1

Fixed effects in the linear mixed models separately for each experiment.

Experiment 1 (16-items sequences, excluding super-deviants)

\begin{tabular}{lccccc}
\hline Predictors & Estimates & Std. Error & T-value & $95 \%$ CI & $p$ \\
\hline (Intercept) & 356.90 & 80.51 & 4.43 & $199.5-514.3$ & $<. \mathbf{0 0 0 1}$ \\
Complexity & 52.15 & 3.84 & 13.60 & $44.6-59.7$ & $<. \mathbf{0 0 0 1}$ \\
Surprise & 6.77 & 11.31 & 0.60 & $-15.4-28.9$ & .55 \\
\hline
\end{tabular}

Experiment 2 (12-items sequences, excluding super-deviants)

\begin{tabular}{lccccc}
\hline Predictors & Estimates & Std. Error & T-value & $95 \%$ CI & $p$ \\
\hline (Intercept) & 852.38 & 124.91 & 6.82 & $608.5-1096.2$ & $<.0001$ \\
Complexity & 24.21 & 6.29 & 3.85 & $11.9-36.5$ & $<. \mathbf{0 0 0 2}$ \\
Surprise & -43.13 & 21.06 & -2.05 & $-84.4--1.9$ & $<.05$ \\
\hline
\end{tabular}

Experiment 3 (8-items sequences)

\begin{tabular}{lccccc}
\hline Predictors & Estimates & Std. Error & T-value & $95 \%$ CI & $p$ \\
\hline (Intercept) & 852.40 & 73.39 & 11.62 & $707.8-997$ & $<.0001$ \\
Complexity & 10.75 & 3.49 & 3.08 & $3.9-17.6$ & $<.003$ \\
Surprise & -32.37 & 5.60 & -5.78 & $-43.3--21.4$ & $<.0001$ \\
\hline
\end{tabular}

Experiment 4 (6-items sequences, pattern 'AAAAAA' excluded)

\begin{tabular}{lccccc}
\hline Predictors & Estimates & Std. Error & T-value & $95 \%$ CI & $p$ \\
\hline (Intercept) & 751.6 & 47.5 & 15.8 & $658.8-844.5$ & $<.0001$ \\
Complexity & 1.4 & 4.4 & 0.3 & $-7.2-9.9$ & .75 \\
Surprise & -15.3 & 3.8 & -4.1 & $-22.7--7.9$ & $<.0001$ \\
\hline
\end{tabular}

Experiment 5 (8-items sequences, auditory and visual)

\begin{tabular}{lccccc}
\hline Predictors & Estimates & Std. Error & T-value & $95 \%$ CI & $p$ \\
\hline (Intercept) & 645.1 & 92.2 & 7.0 & $464.4-825.9$ & $<.0001$ \\
Complexity & 25.2 & 25.2 & 4.4 & $14-36.4$ & $<.0001$ \\
Surprise & -36.7 & 8.1 & -4.5 & $-52.5--20.8$ & $<.0001$ \\
Modality (Visual) & 337.0 & 337.0 & 14.2 & $290.7-383.3$ & $<.0001$ \\
\hline
\end{tabular}

\section{Experiment 2 (12-items long auditory sequences)}

\section{Methods}

\section{Participants}

Twenty healthy volunteers $\left(M_{\text {age }}=26.5, S D=9.5,15\right.$ women $)$ participated in experiment 2 . They all gave written consent to participate and were paid for their participation.

\section{Stimuli}

Auditory binary sequences of twelve sounds were used for this experiment. They were composed of an alternation of the same two complex tones as in the previous experiment, with the same 
duration and SOA. Total sequence duration was $2800 \mathrm{~ms}$. Twelve different sequential patterns, each composed of 6 As and 6 Bs were presented to each participant (Figure 4).

\section{Procedure}

The same procedure and material as in the previous experiment was used. The complexity rating task was performed first (each of the twelve sequences was presented three times, in a pseudo-random order) followed by the violation detection task. In the latter, each sequence was tested in a different short session of approximately 3 min (habituation block of 8 trials, two test blocks of 18 trials each), followed by a pause. Each sequence lasted $2800 \mathrm{~ms}$ and was followed by a $1000 \mathrm{~ms}$ intertrial blank. Order of blocks was randomized for each participant. Half of the trials in tests block contained one deviant tone (at positions 7, 8, 9, 10, 11 or 12): 2/3 of "sequence deviants", 1/3 of "super-deviants". Participants were asked to press the button, as quickly as possible, as soon as they detected that the sequence has been altered.

\section{Data analysis}

The same analysis as in the previous experiment were conducted. Extreme response times were removed (using the same procedure as in experiment 1), and represented $1.2 \%$ of all RTs. One participant was excluded (average number of false alarms per sequence more than $2.5 S D$ above the group median). 
LoT complexity

(A) A A A A B B B B B 6 (A) A A B B A A A B B 6 A B B A A A B B A B 8 (A) A B A A B B A A B 9 (A) A B B A B A B B B 11 (A) A B B B A A B A B 12 A B B A B A A A A B 13 (A) A B B B A A B A B 14 (A) A B B A B A B B B 14 (A) A B A B B A A B B 16 (A) B A A A B B B B B 18 A B B B B A B A A B 19

Figure 4: Twelve 12-item sequences used in experiment 2, with their corresponding LoT complexity value (in bits).

\section{Results and discussion}

\section{Complexity rating task}

A positive linear relationship was found between subjective complexity ratings and LoT complexity $(t(238)=6.81 \mathrm{p}<.0001, r=.61)$. The correlation of the average score per sequence with LoT complexity was however less strong than what was observed in the previous experiment with 16items long sequences $(r=.61$, see Figure 5A). Subjective complexity was clearly underestimated for one specific sequence (“ABBAABABBAAB”, predicted complexity of 8), which is confirmed by an inspection of the residuals of the regression (residual 1.99 SD above average for this sequence).

\section{Deviant type and complexity effects in the violation detection task}

Regarding the violation detection task, main effects of LoT complexity $(t(431.1)=6.43, \mathrm{p}<$ .0001 ) and deviant type (1078 ms for sequence deviants vs. $545 \mathrm{~ms}$ for super-deviants; $t(431.0)=19.3$, $\mathrm{p}<.0001)$ were observed, as well as their interaction $(t(431.1)=3.48, \mathrm{p}<.0006)$. The slope of the 
complexity effect appeared indeed stronger for sequence deviants as opposed to super-deviants (respectively $+30 \mathrm{~ms}$, vs. $+7 \mathrm{~ms}$; see Figure 5B). Separated analyses revealed that it was significant in analyses including either sequence deviants only $(t(205.1)=5.78, \mathrm{p}<.0001 ; r=.63)$, or super-deviants only $(t(208.0)=2.88, \mathrm{p}<.005 ; r=.59)$. The number of false alarms per sequence ( 3.88 on average) was also predicted by the LoT complexity of the sequence $(t(208.0)=3.50, \mathrm{p}<.0006 ; r=.56)$.

As in the complexity rating task, although the overall correlation was high, a noticeable deviation between predicted complexity and observed performance was present for some of the sequences. In fact, the correlation profiles observed in the Figure $5 \mathrm{~A}$ and $5 \mathrm{~B}$ suggest that the psychological complexity of the pattern, as indexed by subjective rating or violation detection task performance, might have been, for some sequences, consistently overestimated or underestimated by the LoT across both tasks (the largest residual in the regression with the sequence deviants, $1.50 \mathrm{SD}$ above average, corresponded to the same sequence identified by complexity ratings : “ABBAABABBAAB”). To test further this idea, we computed the correlation between the residuals of both linear regressions. The correlation was significant $(t(10)=4.02 ; \mathrm{p}<.003)$, indicating that even after regressing out the effect of LoT complexity, the data from both experiments remained correlated with each other, and thus that, although the proposed LoT is a good predictor, it does not fully account for all details of the psychological complexity of patterns. One attempt to address such potential limitations of the language is reported in the Further analysis section. 
A Complexity rating task

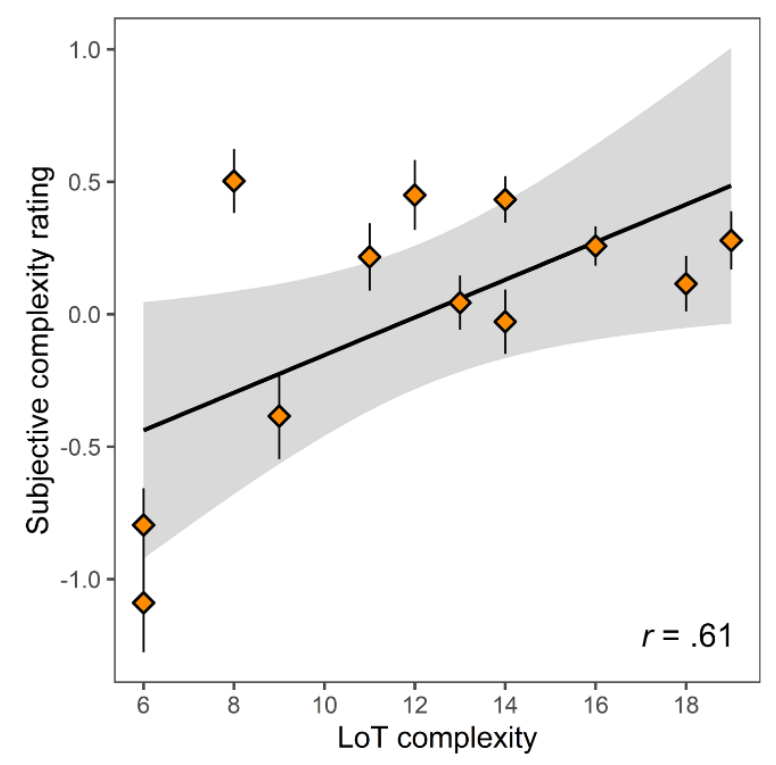

B

Deviant detection task

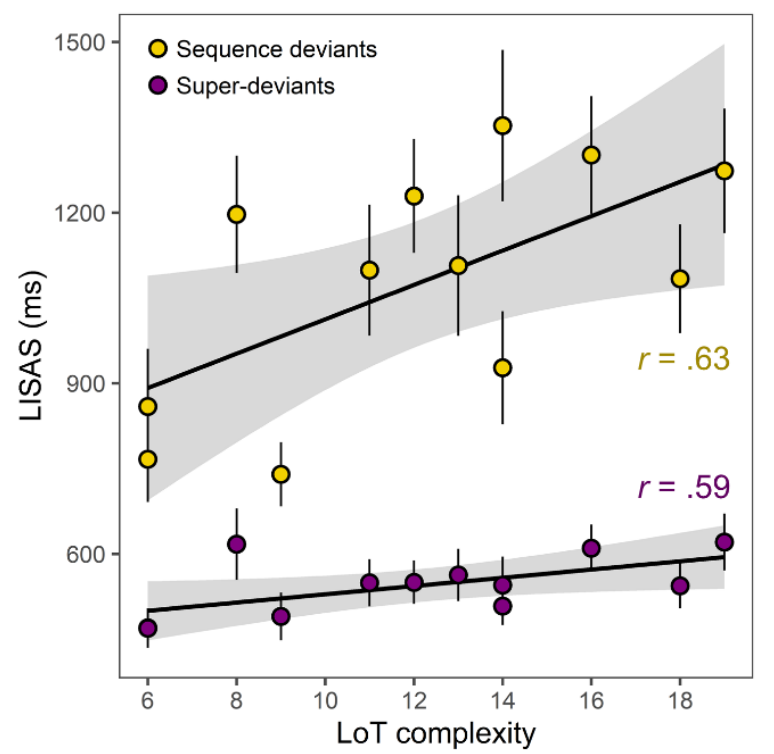

Figure 5: Linear relationship between LoT complexity and scores obtained in the two tasks of experiment 2 with 12-item auditory sequences (with 95\% confidence intervals bands in gray). Same format as Figure 3.

\section{Surprise effects}

A comparison of mixed models (with participants as a random effect) showed that, compared to a null model only including the predicting power of surprise (null model; in which the main predictor was significant: $t(205.0)=4.67, \mathrm{p}<.0001)$, a model also including LoT complexity (full model) fitted the data better (likelihood ratio test : $\chi^{2}(1)=14.4, \mathrm{p}<.0002$ ). Both fixed effects were significant in the full model: LoT complexity $(t(204.1)=3.85, \mathrm{p}<.0001)$, as well as surprise $(t(204.0)=2.05, \mathrm{p}<.05)$ 
(see Table 1). Although we can conclude that the effect of statistical learning (indexed by the level of surprise of deviant items) is here stronger than in the previous experiment (in which it was not clearly significant), note that the effect of surprise remained low.

\section{Experiment 3 and 4 (8-items and 6-items long sequences)}

The main objective of experiments 3 (with 8 -items long sequences) and 4 (with 6-items long sequences) was to test whether the effect of complexity observed in the first two experiments could be generalized to larger sets of shorter sequences, where we could examine more gradual variations in complexity. The same violation detection paradigm was used. No subjective complexity ratings were collected (given the increased number of included sequences compared to the previous experiments).

\section{Methods}

\section{Participants}

Thirty-two healthy volunteers $\left(M_{\text {age }}=27.4, S D=5.3,21\right.$ women $)$ participated in experiment 3 and twenty-three in experiment $4\left(M_{\text {age }}=23.4, S D=4.5,18\right.$ women $)$. They all gave written consent to participate and were paid for their participation.

\section{Stimuli}

In experiment 3, auditory binary sequences of eight sounds were used. They were composed of an alternation of the same two complex tones as in the previous experiment, with the same duration and SOA. Total sequence duration was $1800 \mathrm{~ms}$. Thirty-five different sequential patterns were presented to each participant, i.e. all possible 8-element-long binary combinations that contained the same number of As and Bs (as in experiment 1 and 2).

In experiment 4, auditory binary sequences of six sounds were used (1300 ms). Thirty-two different sequential patterns were presented to each participant, representing all $2^{5}$ types of 6-element 
sequences (given that the labelling of $\mathrm{As}$ and $\mathrm{Bs}$ is arbitrary, sequences such $\mathrm{ABABAB}$ and $\mathrm{BABABA}$ were considered identical). Note that, in this case, the proportion of As vs. Bs varied across sequences.

\section{Procedure}

The same procedure and material as in previous experiments were used. Each sequence was however here tested in a single block of 35 trials (auditory sequence of 1800 or $1300 \mathrm{~ms}$ and inter-trial duration of $1000 \mathrm{~ms}$ ). Alterations of the sequence occur on $1 / 3$ of the trials, starting from the $9^{\text {th }}$ trial (i.e. the habituation phase comprised 8 repetitions). Deviant tones (sounds A replaced by B or conversely — there were no super-deviants in these experiments) were positioned in the second half of the sequence (four or three equiprobable positions). As before, participants were asked to press the button, as quickly as possible, as soon as they detected that the sequence has been altered.

\section{Data analysis}

The same analyzes as in the previous experiments were conducted. Extreme response times that were removed represented $1.6 \%$ of RTs in experiment 3 and $1.6 \%$ in experiment 4 . One participant was excluded in experiment 3 (average number of false alarms per sequence more than $2.5 S D$ above the group median), and one in experiment 4 (average miss rate more than $2.5 S D$ above the group median).

\section{Results and discussion}

Here again, we tested (using mixed models) whether surprise suffices to explain the variance in performance or if a significant proportion remained yet to be explained by sequence complexity (all models included participants as a random effect). In experiment 3 ( 8 -items sequences, $\mathrm{N}=35$ ), goodness of fit improved when LoT complexity was included in the model $\left(\chi^{2}(1)=9.47, \mathrm{p}<.003\right)$. Both fixed effects were significant in the full model: LoT complexity $(t(1042.0)=3.08, \mathrm{p}<.003$; see Figure 6A), as well as surprise $(t(1042.0)=5.78, \mathrm{p}<.0001)$ (see Table 1$)$. Note that the surprise fixed effect was already highly significant in the null model $(t(1043.0)=8.72, \mathrm{p}<.0001)$.

Similarly, in experiment 4 (6-items sequences, $N=32$ ), goodness of fit improved when LoT complexity was included to the surprise-only null model $\left(\chi^{2}(1)=6.20, p<.02\right)$ with both fixed effects 
significant in the full model (LoT complexity: $t(649.00)=2.49, \mathrm{p}<.02$; see Figure $6 \mathrm{~B}$ ), and surprise $(t(649.0)=5.48, \mathrm{p}<.0001)$. The surprise fixed effect was here again already highly significant in the null model $(t(650.0)=6.78, \mathrm{p}<.0001)$. However, one sequence appeared as an outlier in this experiment, with an average LISAS 3.9 SD below the average of all sequences (i.e. indicating a much better performance): the "AAAAAA" sequence. In this case, performing the task requires no sequence learning, but merely remembering the identity of the A sound, and violation detection is therefore similar to a classic oddball paradigm. When this sequence was removed from the dataset (it was also excluded from further analyses), the inclusion of the complexity fixed factor did no longer improved model goodness of fit $\left(\chi^{2}(1)=0.10, p=.75\right)$. Indeed, the LoT complexity fixed effect was not significant in the full model $(t(628.0)=0.32, \mathrm{p}=.75)$, as opposed to the surprise fixed effect $(t(628.0)=4.07, \mathrm{p}<.0001)$ (see Table 1). No improvement in model fit was found when including the interaction between complexity and surprise $\left(\chi^{2}(1)=0.08\right.$ in experiment $3, \chi^{2}(1)=0.34$ in experiment 4$)$.

Beside the effect of complexity, the strong effect of surprise in both experiments indicates that participants were quicker and more likely to detect a deviant when it violated statistical regularities characterizing the auditory sequence being repeatedly played. This is consistent with the idea that humans spontaneously encode the probabilities associated with events and react to surprising events depending on their level of predictability (Huettel et al., 2002; Meyniel et al., 2016).

The number of false alarms was low in the present experiments ( 0.91 per sequence on average in experiment 3,0.60 in experiment 4). It was slightly related to sequence complexity in experiment 3 $t(1048)=2.19, \mathrm{p}<.03)$ but not in experiment $4 t(650.0)=0.29, \mathrm{p}=.77)$

Compared to the previous experiment with lengths 12 and 16, it was expected here, with sequences of 8 or 6 items, that the effect of LoT complexity would be mitigated, since those auditory sequences may become short enough to be stored in working memory as a simple chain (note that the range of LoT complexity values was also smaller). The correlation of performance with LoT complexity was in fact still present with 8-items sequences (at a similar level as in experiment 2) but disappeared with 6-items sequences. This is in line with the assumption that complexity is tightly linked with the idea of compressibility in memory, and suggests that such a compression strategy is more likely to be 
involved when the number of items to store in working memory exceeds the typical working memory span. However, rather than a clear threshold above which complexity would become predictive of performance, the estimates of the LoT complexity effect across the four experiments (in the mixed models taking into account surprise) reveal a gradient: with stronger effects of complexity for longer sequences (respectively $+1.4 \mathrm{~ms},+10.8 \mathrm{~ms},+24.2 \mathrm{~ms}$, and $+52.2 \mathrm{~ms}$, for the experiments with length 6 , 8, 12 and 16 respectively; see Table 1). The effect of surprise seemed to follow an inverse trend, with insignificant or marginal effects in long sequences (experiments 1 and 2) and highly significant effects in short sequences (experiments 3 and 4). To test this idea, the data from experiments 1-4 (excluding super-deviants) were combined in a single mixed model including the three fixed factors of LoT complexity, surprise and length (as a continuous predictor), as well as the three two-way interactions (with participants as the random factor). An ANOVA on the mixed model revealed main effects of LoT complexity $(F(1,2336.4)=48.0, \mathrm{p}<.0001)$ and surprise $(F(1,2334.1)=4.91, \mathrm{p}<.03)$. The main effect of sequence length was marginally significant $(F(1,96.6)=3.08, \mathrm{p}=.082)$. As expected, a strong interaction between LoT complexity and length was present $(F(1,2347.5)=63.3, \mathrm{p}<.0001)$, indicating a stronger effect of complexity when sequence length increased. The estimated slopes for the LoT complexity effect indeed increased with each sequence length $(+15.5 \mathrm{~ms},+46.0 \mathrm{~ms},+107.1 \mathrm{~ms}$, and $+168.1 \mathrm{~ms}$, for length $6,8,12$ and 16 , respectively). The interaction between length and surprise was not significant $(F(1,2330.0)=1.19, \mathrm{p}=.28)$. However, the estimated slopes for the surprise effect followed our initial observation: they decreased with each sequence length $(-15.6 \mathrm{~ms},-12.0 \mathrm{~ms},-4.9 \mathrm{~ms}$, and $+2.2 \mathrm{~ms})$. 

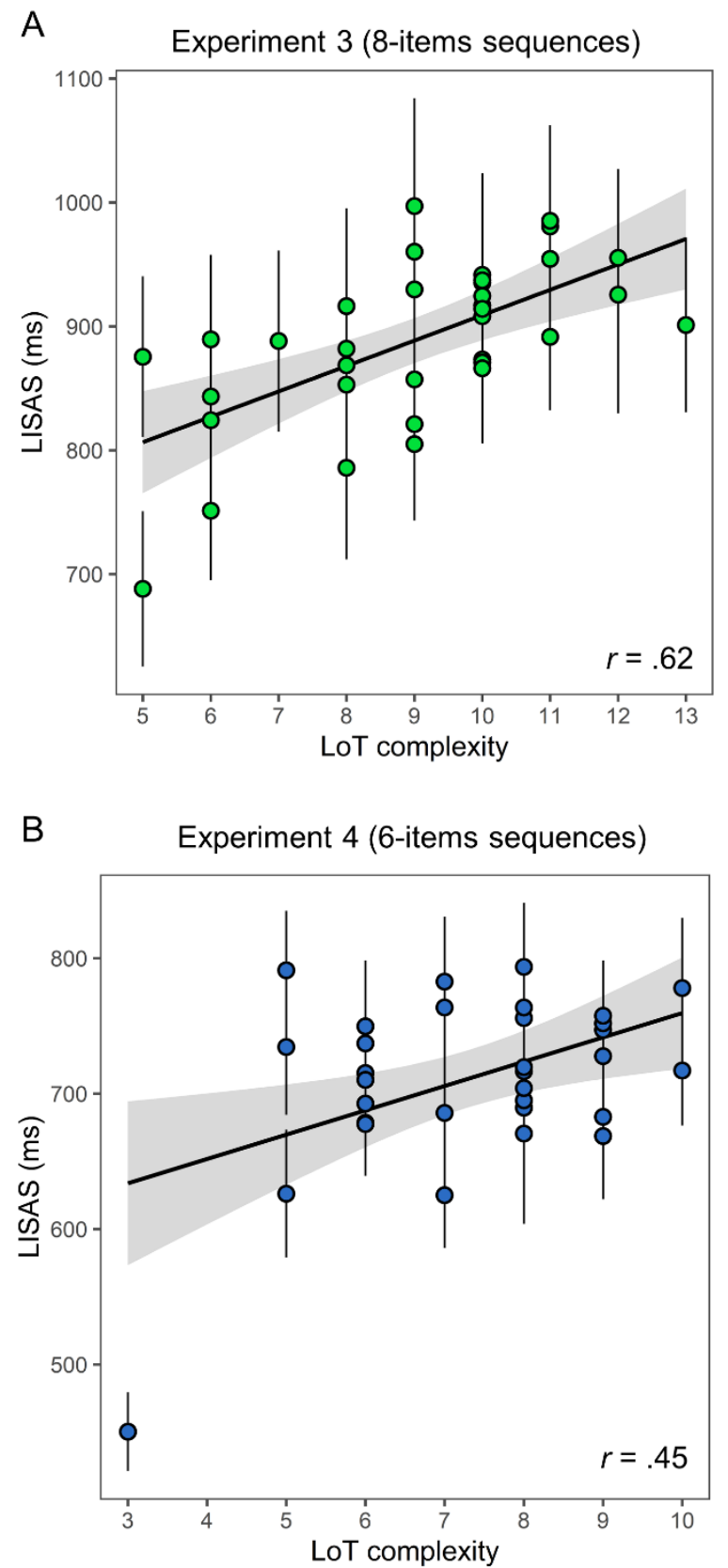

Figure 6: Linear relationship between LoT complexity and violation detection task performance (LISAS) in: (A) experiment 3 (8-items sequences) and (B) experiment 4 (6-items sequences).

\section{Experiment 5 (auditory vs. visual modality)}

The observation of a LoT complexity effect on sequences of length 8 and higher is consistent with our initial claim that individuals spontaneously apply simple rules (mainly based on nested repetitions) in order to recode auditory sequences in a compressed abstract form in memory. It may be 
argued, however, that rather than being abstract and universal, some of these effects may reflect the great ability of our auditory system to manipulate and find regularities in acoustic stimuli; whether it is in spoken language or in music listening. In experiment 5, we wished to replicate the findings of previous experiment and extend them to the visual modality. Sequences of 8 items (allowing to use a sufficient number of trials while still expecting clear complexity effects) were presented to a group of participants in both a visual and in an auditory form (in different experimental blocks), using the same violation detection paradigm. Due to constraints in the perception of repeated visual stimuli, stimulus onset asynchrony was lengthened to $400 \mathrm{~ms}$ in both auditory and visual sessions, resulting in a sequence duration of $3000 \mathrm{~ms}$ (compared to $1800 \mathrm{~ms}$ in experiment 2).

\section{Methods}

\section{Participants}

Participants were eighteen healthy volunteers $\left(M_{\text {age }}=25.5, S D=5.7,15\right.$ women $)$. They all gave written consent to participate and were paid for their participation.

\section{Stimuli}

Fifteen binary sequential patterns of eight items were used for this experiment (all were composed of 4 items A and 4 items B). All were previously used in experiment 2 . They were selected based on their LoT complexity, in order to preserve a large and homogenous distribution of complexity values. The same sequences were presented to participants in auditory and visual forms (in different blocks). Auditory sequences were composed of the same two complex tones as in the previous experiments. Visual sequences were composed of two colored Gabor patches presented in the center of the screen (a red Gabor patch with $45^{\circ}$ orientation, and a green patch with $135^{\circ}$ orientation). Stimulus duration was $200 \mathrm{~ms}$ with $200 \mathrm{~ms}$ inter-stimulus interval in both modalities. Total sequence duration was $3000 \mathrm{~ms}$.

\section{Procedure}


The same procedure and material as in previous experiments were used in the auditory blocks. Participants were instructed to fixate the center of the screen in the visual blocks. Each sequence was tested in a short block of approximately $2.5 \mathrm{~min}$., followed by a pause. Since each sequence was presented twice (i.e. in the visual and in the auditory form), the experiment was divided in two sessions of fourteen blocks, separated by a longer pause. Each pattern appeared once in a given session, which comprised equal numbers of auditory and visual blocks. Order of blocks within each session was randomized for each participant. Each block comprised 35 trials (sequence of $3000 \mathrm{~ms}$ and inter-trial duration of $1000 \mathrm{~ms})$. The habituation phase contained at least eight trials, alterations of the sequence occur on $1 / 3$ of the remaining trials (i.e., 9 deviant trials). As before, deviant items only appeared within the second half of the sequence (positions 5, 6, 7 or 8). Participants were asked to press the button, as quickly as possible, as soon as they detected a change in the sequence.

\section{Data analysis}

LISAS were computed for each sequence per modality per subject using correct response times and miss rate (after removing $2.4 \%$ extreme response times). One participant was excluded (miss rate and number of false alarms more than $2.5 S D$ above group median). The same analysis procedure described before was adopted with the sole exception that some analyses included modality as a categorical two-levels (auditory vs. visual) predictor.

\section{Results and discussion}

\section{Complexity and modality effects}

To assess the impact of LoT complexity and modality on performance, we first computed a mixed model including complexity and modality as fixed factors and participants as a random factor. Effects of LoT complexity $(t(486.0)=3.08, \mathrm{p}<.003)$, modality (average LISAS of $1110 \mathrm{~ms}$ in visual blocks vs. $780 \mathrm{~ms}$ in auditory blocks; $t(486.0)=14.1, \mathrm{p}<.0001)$ and their interaction $(t(486.0)=3.19$, $\mathrm{p}<.002)$ were significant. The slope of the complexity effect was steeper in the visual than in the auditory modality $(+54 \mathrm{~ms}$ vs. $+22 \mathrm{~ms}, t(486)=3.19$; see Figure 7$)$. Separate analyses indicated that 
LoT complexity was a strong predictor of performance for visual sequences $(t(233.0)=6.82, \mathrm{p}<.0001$; $r=.76)$, and also for auditory sequences $(t(237.0)=3.76, \mathrm{p}<.0003 ; r=.63)$.

Note that, although the effects appeared stronger for the visual modality, the average performance in the visual and the auditory modality were highly correlated $(r=.85, \mathrm{p}<.0001)$. This suggests a common, cross-modal mechanism behind the observed differences in performance between sequences. It can however be acknowledged, here again, that this is not fully explained by complexity. Indeed, the residuals of linear regressions with LoT complexity in the visual and in the auditory modality (using average LISAS per sequence) were still correlated $(r=.73, t(13)=3.92 ; \mathrm{p}<.002)$.

The number of false alarms per sequence was related to the task modality (mean number of FA: 0.58 in auditory blocks; 1.16 in visual blocks; difference between modalities: $t(487.0)=5.73, \mathrm{p}<.0001$ ) but not to sequence LoT complexity $(t(487.0)=0.08, \mathrm{p}=.94)$.

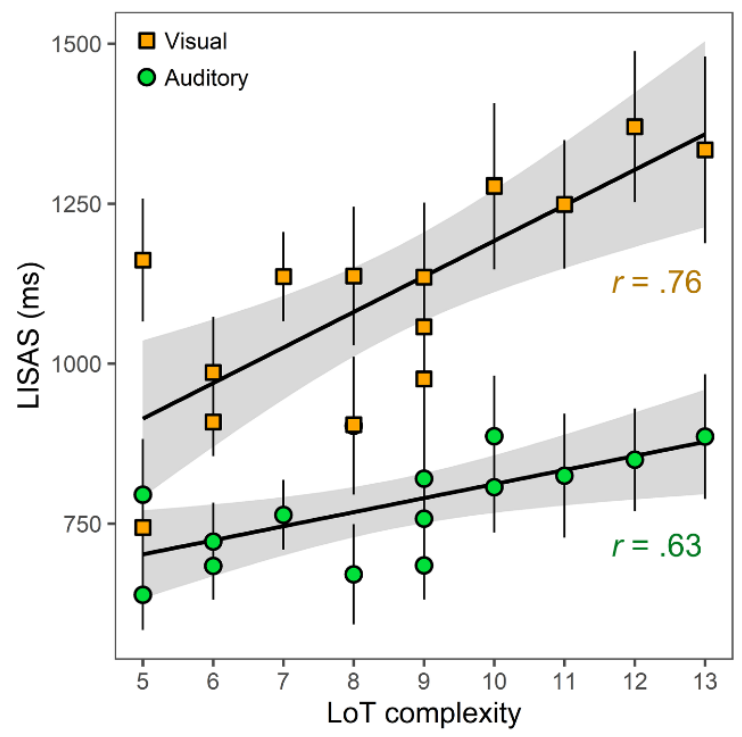

Figure 7: Linear relationship between LoT complexity and violation detection task performance (LISAS) for each modality in experiment 5 (8-items auditory and visual sequences).

\section{Surprise effects}

As in previous experiments, a surprise effect was also observed, in both modalities, when considered independently; deviants inducing rare transitions were more easily and quickly detected than frequent ones (effect of surprise in a mixed model with auditory trials only: $t(237.0)=3.87, \mathrm{p}<.0002$; 
$r=-.65$; with visual trials only: $t(233.0)=6.79, \mathrm{p}<.0001 ; r=-.78)$. This effect suggests that a common, or at least a similar, mechanism is at play in the encoding of statistical regularities characterizing the sequences in both the visual and the auditory modality.

In order to test whether evidence for sequence compression could still be observed once the surprise effect was taken into account, we performed a comparison of mixed effects models. The null model included the surprise predictor, the modality as a categorical predictor and the subject random factor. It was compared against a full model including the same predictors, with the addition of the LoT complexity. This comparison was highly significant $\left(\chi^{2}(1)=19.0, p<.0001\right)$, indicating that goodness of fit improved when LoT complexity was added to the model. All three fixed effects were significant in the full model (LoT complexity: $t(486.0)=4.39, \mathrm{p}<.0001$; surprise: $t(486.0)=4.54, \mathrm{p}<.0001$; modality: $t(486.0)=14.2, \mathrm{p}<.0001$, see Table 1$)$

Overall, the results obtained in the visual modality are very similar to those obtained in the auditory modality in the same and in previous experiments. We however observed here stronger effects of both LoT complexity and surprise. It should be noted that the overall difficulty of the task increased in the visual modality (as indicated by higher average miss rates per sequence; $22 \%$ vs. $11 \%, t(14)=$ $7.49, \mathrm{p}<.0001$; and longer average response times per sequence; $831 \mathrm{~ms}$ vs. $645 \mathrm{~ms}, t(14)=10.5, \mathrm{p}<$ .0001). 8-items visual sequences may have been more difficult to encode than 8-items auditory sequences, due to the known superiority of the auditory processing system in the processing of temporal sequences and rhythms (Freides, 1974; Patel, Iversen, Chen, \& Repp, 2005). This increased encoding difficulty in the visual domain may have in turn lead to an increased need for the "mental sequence compression" mechanism that our language of thought aims to describe.

The present experiment also extends the results of experiment 3 by using a slower presentation rate. Indeed, although the participants in experiment 5 appeared to respond faster (in the auditory blocks) than those from experiment 3 , the same relationship with complexity was found (correlation of performance with LoT complexity of .62 and .63 respectively). It suggests that the effect of complexity is robust across sequence durations (as expected given than LoT complexity is based on abstract 
sequence patterns). More importantly, the fact that a similar complexity effect was observed irrespective of the modality is consistent with the idea of "language of thought" used to compress sequential information at an abstract, symbolic level. Such an assumption has already been supported by results from Yildirim and Jacobs (2015), who showed cross-modal transfer of sequence knowledge: learning to categorize visual sequences facilitated the categorization of auditory sequences and vice versa. In fact, the language we used here was initially designed to represent visually presented, geometrical patterns (Amalric et al., 2017). The present results thus confirm that this language can account for sequence representations in various modalities and presentation contexts.

\section{Further analysis: comparison with other measures of}

\section{sequence complexity}

Several measures of sequence complexity have been previously proposed in the psychological literature (Aksentijevic \& Gibson, 2012; Alexander \& Carey, 1968; Gauvrit et al., 2014; Glanzer \& Clark, 1963; Griffiths \& Tenenbaum, 2003; Psotka, 1975; Vitz, 1968; Vitz \& Todd, 1969). In this last section, we examined how our LoT complexity value compares to five other measures in predicting task performance over different sequence lengths. These measures were the following:

The number of runs of identical items (or "chunks", defined as consecutive repetitions of the same item), a rough measure of complexity previously shown to be correlated to performance in sequence encoding tasks (e.g., Glanzer and Clark, 1963).

Entropy: a measure of information that quantifies the uncertainty of a distribution. Here we computed the Shannon entropy $(H)$ of the probability of pairs of items (AA, AB, BA, BB), in order to capture the effect of order-1 transition probabilities (Meyniel et al., 2016). Given that the probability of a given pair is defined as $p(X, Y)=p(X) \cdot p(Y \mid X), H$ is computed as follow:

$$
\begin{gathered}
H=-\left[p(A) \cdot p(A \mid A) \cdot\left(\log _{2} p(A)+\log _{2} p(A \mid A)\right)+p(A) \cdot p(B \mid A) \cdot\left(\log _{2} p(A)+\log _{2} p(B \mid A)\right)+p(B)\right. \\
\left.\cdot p(A \mid B) \cdot\left(\log _{2} p(B)+\log _{2} p(A \mid B)\right)+p(B) \cdot p(B \mid B) \cdot\left(\log _{2} p(B)+\log _{2} p(B \mid B)\right)\right]
\end{gathered}
$$


We used the convention that $0 * \log _{2}(0)=0$ when null probabilities occurred.

The number of subsymmetries: the number of symmetric sub-sequences within a sequence, proposed by Alexander and Carey (1968), and shown to be negatively correlated to performance in perception and production tasks with visual and auditory patterns (Alexander \& Carey, 1968; Toussaint \& Beltran, 2013).

Change complexity: an advanced measure proposed by Aksentijevic and Gibson (2012), based on the notion of "change" (the inverse of invariance), computed across all sub-sequences contained in a sequence, and showing interesting properties such as a sensibility to periodicity and symmetries.

Algorithmic complexity, introduced by Gauvrit et al., $(2014,2016)$ and Soler-Toscano et al., (2014) based on the mathematical definition of Kolmogorov-Chaitin complexity (Chaitin, 1969; Kolmogorov, 1968), and derived from the probability of obtaining a given pattern an output of a Universal Turing Machine that halt.

Note that the alternative measures of complexity tested here, which provide a unique metric for each pattern, are quite conceptually different from the one we advocate for in the present study. LoT complexity is based on the proposal that humans possess a language of thought, composed of a small number of atomic rules which they use recursively to recode the abstract structure of the pattern in a compressed form. Such a recursive representation differs radically from, say, the mere counting of the number of chunks. However, it is possible to combine the two ideas. The formal language we proposed produces many licit expressions for each sequence (the number of possible expressions can reach several tens of thousands for a sequence of length 16), which correspond to distinct "parses" of the same sequence. We initially assumed that the shortest expression is always selected, and thus that LoT complexity is equal to minimal description length (MDL), but it could be that additional filters are imposed. One likely filter is whether the proposed parsing coincides with chunk boundaries. Consider the sequence "ABBAAB", which consists of 4 chunks $[A][B B][A A][B]$. According to our language, its optimal description is $[\mathrm{AB}][\mathrm{BA}][\mathrm{AB}]$ (i.e. 3 repetitions of the stay-change program; LoT complexity =5). Interestingly, the data suggested that MDL may not be optimal in similar cases (see Experiment 2, Results and discussion). One possibility is that participants begin by parsing the sequence into chunks 
before searching for a minimal expression, and therefore the MDL is only computed over expressions whose parsing coincides with chunk boundaries. To test this idea, we recomputed LoT values restricted to chunk-preserving expressions (i.e., excluding expressions producing “A][A" or "B][B"). With such a constraint, this new LoT complexity (hereafter called LoT-chunk) was higher than the original one for $60 \%$ of sequences (and remained the same otherwise). For instance, the sequence "ABBAAB" from the previous example, when described as four chunks [A] [BB] [AA] [B], has an LoT-chunk complexity $=9$. We tested LoT chunk complexity as another potential predictor of behavioral performance.

To conduct the analyses, data were pooled from all previous experiment with auditory sequences (using LISAS to index task performance), excluding super-deviants trials. Unfortunately, due to the nature of algorithmic complexity (derived from the output frequency for a pattern using small Turing machines, which decreases rapidly with sequence length), no values were available for the ten length16 patterns that we used in experiment 1 , as well as for one length- 12 pattern used in experiment 2 . Those sequences were therefore excluded from some analyses. The sequence "AAAAAA" from experiment 4 was also excluded. Consequently, a first pooled dataset, for which all 7 different predictors could be compared, included performance with 77 different auditory sequences (and 88 different participants), of length $6(n=31$ sequences $)$, length $8(n=35)$ and length $12(n=11)$, while a second one, for which 6 different predictors were compared, also included sequences of length 16 ( $\mathrm{n}=88$ sequences, 113 participants).

To assess whether one measure was a better predictor of task performance, we first computed different mixed models, which all included the predictor of interest as the only fixed effect and participants as a random effect (note that this is a way to control for the fact that different participants coming from different experiments, with different sets of stimuli, were pooled together). We then report the Akaike information criterion (AIC) as an indicator of goodness of fit which penalizes for model complexity (i.e. the number of predictors); the model with the lowest AIC value being considered the best (or with lowest " $\Delta$ (AIC)" value, i.e. the relative difference in AIC with the best model: for a model $\left.i, \Delta(\mathrm{AIC})_{\mathrm{i}}=\mathrm{AIC}_{\mathrm{i}}-\mathrm{AIC}_{\mathrm{min}}\right)$. Note that we also report the Bayesian information criterion (BIC) which, in addition, scales the strength of penalization by the $(\log )$ number of data points. Second, since, as we 
reported earlier, surprise derived from the learning of transition probabilities may strongly affect the performance in such violation detection task, all these models were computed again, this time including surprise as a fixed effect covariate.

\section{Dataset with sequences of length 6,8 and 12 .}

Fourteen different mixed models were fitted using datasets with sequences of length 6,8 and 12. As illustrated in Figure 8A, model fit (as indexed here by the $\Delta$ (AIC) value) always improved when the surprise associated to the deviants was included in the model. This finding confirms that the effect due to transition probabilities needs to be taken into account when assessing responses to deviants in the violation detection paradigm. When considering either only single-predictor models (i.e. without the surprise covariate) or two-predictors models (i.e. with surprise), the two best models were the ones with our modified version of LoT complexity (i.e. LoT-chunk, with the "no-splitting" chunks constraint) followed by the one with original LoT complexity (see Table 2). In order to test whether the differences in the raw AIC values were meaningful, we computed the Akaike weights for this set of 14 models. Akaike weights can be interpreted as the probability that a given model is the best model of the set (Wagenmakers \& Farrell, 2004). Akaike weight was .99 for the LoT-chunk complexity (+ surprise) model, .01 for the LoT complexity (+ surprise) model, and below .01 for all other models (see Table 2 and Figure 8A). 

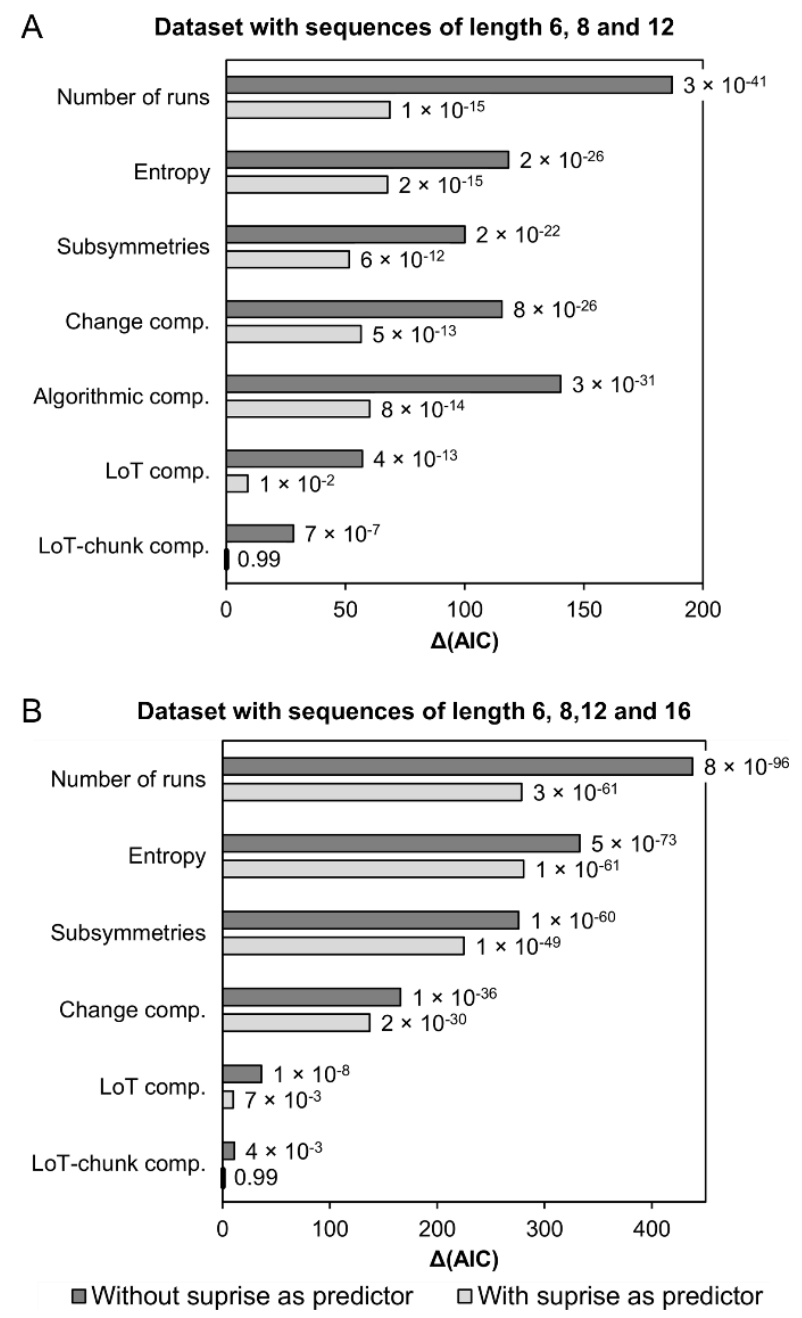

Figure 8: $\Delta$ (AIC) for the fourteen mixed models tested using the dataset including the task performance (LISAS) for sequences of length 6,8 and 12 (A), and for the ten different mixed models tested using the dataset with sequences of length $6,8,12$ and 16 (B). The fixed effect of interest is indicated along the vertical axis (all models included participants as a random effect and could include surprise as a covariate — light gray bars). Akaike weight for each model is also reported. The model with lower $\mathrm{AIC}(\Delta(\mathrm{AIC})=0)$ is indicated by short dark vertical line on the vertical axis.

Although the correlations of performance with LoT complexity in experiments 2, 3 and 4 (lengths 6, 8 and 12) were small in comparison with experiment 1 (length 16), LoT complexity again appears as the best predictor of performance in the violation detection task with sequences of length $\leq$ 12. Notably, the constraint of excluding, for each pattern, the expressions that resulted in the splitting of a chunk (before the selection of shortest expression) improved the fit to the behavioral data. This observation suggests that participants did not always find the best way of coding some patterns (best in the sense of the language of thought considered here) because of a propensity to perform an initial chunking solely based on consecutive runs of identical items. It would however be premature to draw 
strong conclusions about a general mechanism since improvement in goodness of fit was modest considering the $\Delta$ (AIC) of the other models, and not necessarily true for each individual experiment and individual participant.

The next best model was the one with the "number of subsymmetries" predictor (and including the surprise covariate), suggesting that it also provides a good measure of the psychological complexity of patterns. However, while this appeared true here using statistical models partially controlling for sequence length (i.e. by including participant index as a random factor, since each participant performed the task with only one given sequence length), this measure appears inappropriate to predict complexity across different lengths. Indeed, when we computed the Pearson correlation of average LISAS per sequence for the pooled dataset (sequences of length 6,8 and 12), we obtained a positive correlation value of .39. Such positive correlation is in conflict with the presupposition that patterns containing more symmetries should be simpler. It is due to the fact that the number of subsymmetries tends to increase with sequence length. These correlations were actually negative when each length was considered independently ( $r=-.44$ for length $6 ; r=-.54$ for length 8 ; and $r=-.58$ for length 12 ; see additional linear regression lines of Figure 9). To summarize, although this measure is quite good in predicting the complexity of sequences of a given length, it is not efficient in predicting the increase in complexity due to the lengthening of the pattern to encode. LoT complexity, on the other hand, nicely captures complexity variations due to both structure and length.

Another similar limitation can also apply to algorithmic complexity, where the correlation observed across lengths $(r=.79)$ is mostly because this value presents excessive discontinuities with length: algorithmic complexity ranges roughly between 14 and 16 for length 6; between 19 and 23 for length 8; and between 31 and 35 for length 12 (see Figure 9). These large increases in complexity with length are not consistent with behavior. Again, LoT complexity, for which some 6-items long patterns can be more complex than some 12-items ones, provides a better correlation with the present behavioral data. 
Table 2

Model comparisons for the each of the two datasets

\begin{tabular}{lcccccccc}
\hline & \multicolumn{3}{c}{ Dataset with sequences of length 6, 8 and 12 } & \multicolumn{3}{c}{ Dataset with sequences of length 6, 8, 12 and 16 } \\
Model fixed effect $(s)$ & Log-lik. & $\Delta(\mathrm{AIC})$ & $\Delta(\mathrm{BIC})$ & $\mathrm{w}(\mathrm{AIC})$ & Log-lik. & $\Delta(\mathrm{AIC})$ & $\Delta(\mathrm{BIC})$ & $\mathrm{w}(\mathrm{AIC})$ \\
\hline LoT comp. & -14886 & 57 & 51 & $4.0 \times 10^{-13}$ & -16653 & 36 & 31 & $1.3 \times 10^{-8}$ \\
LoT comp. + Surp. & -14861 & 9 & 9 & $1.1 \times 10^{-2}$ & -16639 & 10 & 10 & $6.8 \times 10^{-3}$ \\
LoT-chunk comp. & -14872 & 28 & 23 & $7.4 \times 10^{-7}$ & 33444 & 11 & 5 & $4.2 \times 10^{-3}$ \\
LoT-chunk comp. + Surp. & -14857 & 0 & 0 & 0.99 & 0 & 0 & 0 & 0.99 \\
Number of runs & -14951 & 187 & 181 & $2.5 \times 10^{-41}$ & -16854 & 438 & 432 & $7.9 \times 10^{-96}$ \\
Number of runs + Surp. & -14891 & 69 & 69 & $1.3 \times 10^{-15}$ & -16773 & 279 & 279 & $3.0 \times 10^{-61}$ \\
Entropy & -14917 & 118 & 113 & $1.9 \times 10^{-26}$ & -16801 & 333 & 327 & $5.4 \times 10^{-73}$ \\
Entropy + Surp. & -14888 & 68 & 68 & $2.0 \times 10^{-15}$ & -16774 & 280 & 280 & $1.3 \times 10^{-61}$ \\
Subsymmetries & -14888 & 100 & 94 & $1.8 \times 10^{-22}$ & -16773 & 276 & 270 & $1.2 \times 10^{-60}$ \\
Subsymmetries + Surp. & -14883 & 52 & 52 & $6.2 \times 10^{-12}$ & -16746 & 225 & 225 & $1.5 \times 10^{-49}$ \\
Change comp. & -14916 & 116 & 110 & $7.6 \times 10^{-26}$ & -16718 & 166 & 160 & $1.0 \times 10^{-36}$ \\
Change comp. + Surp. & -14885 & 57 & 57 & $5.3 \times 10^{-13}$ & -16703 & 137 & 137 & $1.7 \times 10^{-30}$ \\
Algorithmic comp. & -14928 & 140 & 135 & $3.4 \times 10^{-31}$ & & & $N . A$. & N.A. \\
Algorithmic comp. + Surp. & -14887 & 60 & 60 & $8.4 \times 10^{-14}$ & & & \\
\hline
\end{tabular}

Note. All models included participants as a random effect, and either one or two fixed effect(s) (i.e. “+ Surp.”: with additional surprise fixed effect). Log-lik. $=\log$ of the maximum likelihood for the model. $\Delta($ AIC $)=$ AIC difference with the model with the lowest AIC value (where AIC is the Akaike Information Criterion). $\Delta(\mathrm{BIC})=\mathrm{BIC}$ difference with the model with the lowest $\mathrm{BIC}$ value (where BIC is the Bayesian Information Criterion). $\mathrm{w}(\mathrm{AIC})=$ Akaike weight.

\section{Dataset with sequences of length 6, 8, 12 and 16 .}

Twelve different mixed models (with participants as a random effect) were here fitted, using the same dataset as before to which was added data from 11 sequences for which algorithmic complexity value was not available (thus now with sequences of length $6,8,12$ and 16). The same predictors as above were used, with the exception of algorithmic complexity. Here again, as illustrated in Figure 8B, goodness of fit systematically increased when surprise was included. LoT-chunk complexity and LoT complexity (with or without surprise as a covariate) were again the best predictors of performance (see Table 2). As opposed to the previous set of analyses in which the data from experiment 1 (length 16) was not included, the model with change complexity performed clearly better than the one with the number of subsymmetries. The long sequences used in experiment 1 indeed presented important differences in their number of subsymmetries (e.g., 56 for $(A B)^{8}$ vs. 32 for $\left.\left(A^{4} B^{4}\right)^{2}\right)$, which were clearly not predictive of performance. Consequently, and as stated earlier, the number of subsymmetries does not appear as a good predictor of task performance across different sequence lengths. Change 
complexity also appeared as a much better predictor when performing a simple linear regressions on average LISAS per sequence (see Figure 9 for an overview of such linear regression performed with each predictor of interest), resulting in an $r=.81$, which is close to the one obtained with LoT complexity $(r=.82)$. It indicates that change complexity can also be a good measure of the psychological complexity of a sequence regardless of its length. It must however be noted that, contrary to the mixed models, these linear regressions using data averaged over participants did not control for the variance accounted for by surprise, or due to inter-subject variability. Important variations were indeed observed across participants regarding the correlation with complexity (especially for experiments with shorter sequences). When computed at the level of individual participants, the correlation with LoT complexity appeared on average stronger (mean $r=.31, S D=.32$ ) than the one with change complexity (mean $r=$ $.23, S D=.30)(\mathrm{t}(112)=3.54, \mathrm{p}<.0006)$. 

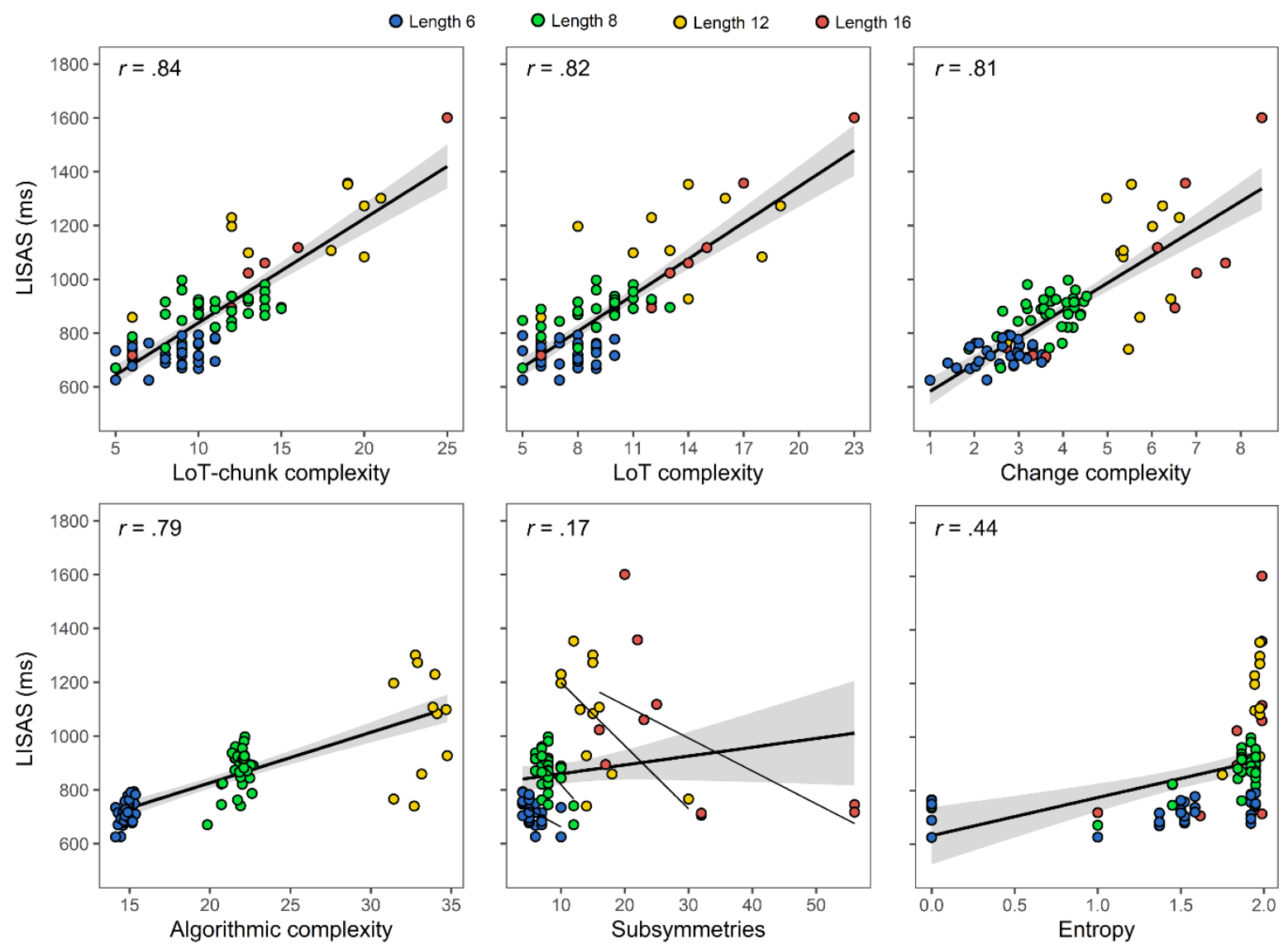

Figure 9: Linear regressions of average performance per sequence (LISAS, in ms) with five different predictors of interest when combining data from experiments with auditory sequences of 4 different lengths. Each marker corresponds to one sequence. Sequences of different lengths are indicated by different markers only for illustration purposes (the length factor was not taken into account when computing the correlation, $r$, coefficient). 16-items long sequences (as well as one 12-items sequence) could not be included in the regression with algorithmic complexity. Regressions lines for each sequence length were added in the subsymmetries plot, in order to illustrate the fact that negative correlations were observed when each length was considered separately. 


\section{General discussion}

The main goal of this series of experiments was to evaluate the mental representation of binary sequences and to test the adequacy of a formal language of thought previously proposed to account for geometrical sequences (Amalric et al., 2017). Across five different experiments with sequences of different lengths, in the auditory but also in the visual modality, we found consistent evidence that a significant part of the variations in sequence encoding performance (as indexed by the capacity to detect sequence violations) was explained by the minimal description length of the sequence in this formal language. These results are consistent with the idea that upon hearing or seeing a binary sequence, subjects form an internal representation corresponding to an abstract and compressed form of the sequence content. It is remarkable that a language merely composed of two simple instructions (repetition and change) and their recursive embeddings suffices to model the formation of such a representation. The complexity measure derived from this language was indeed better predictive of the degree of psychological complexity than other sophisticated approaches designed as alternatives to the non-computable Kolmogorov complexity (Aksentijevic \& Gibson, 2012; Soler-Toscano et al., 2014).

The assumption that minimal description length in the formal language corresponds to perceived sequence complexity was further corroborated by subjective complexity rating (experiments 1 and 2 ). Moreover, we found that sequence structure was not the only information encoded by the participants, since the level of surprise derived from the statistical estimation of transition probabilities also helped explain the variance in violation detection performance. The effects of surprise and of complexity on responses to violations were found to vary differently depending on sequence length, thus providing new insights on how the human brain makes predictions in temporal sequences.

The predictive power of the LoT approach was most notable for the longest sequences tested, in particular for 16 items long sequences (experiment $1 ; r=98$ ). Indeed, massive differences in miss rates were observed between the sequences predicted to be the least complex $\left(\mathrm{A}^{n} \mathrm{~B}^{n}\right.$ patterns, with LoT complexity $=6$ ) and those predicted to be the most complex (a set of 10 instructions, LoT complexity = 23), suggesting that subjects simply could not learn the latter efficiently, even after eight or more 
repetitions. An additional prediction of LoT was verified, namely the fact that the four sequences based on the $\mathrm{A}^{n} \mathrm{~B}^{n}$ pattern were associated with a similar performance level, regardless of $n(=1,2,4$, or 8$)$. In the language, this is due to the fact that the complexity of a repetition is proportional to the logarithm of the number of repetitions, rounded up to the nearest integer. For a total number of 16 items, it therefore does not matter when the sequence is decomposed in 2 chunks of 8,4 chunks of 4,8 chunks of 2 , or 16 chunks of 1: the sum of the weights remains unchanged, leading to a LoT complexity of 6 bits in all cases — and indeed, the observed performance remained stable across such a broad variation ranging from huge chunks to pure alternation (see Figure 3).

The correlation of performance with LoT complexity decreased in subsequent experiments using increasingly shorter sequences, until it became almost absent for sequences comprising only six elements. Rather than an indication of an intrinsic limitation of the language for describing very short binary patterns, we believe that a significant part of this effect relates to differences in working memory demands. The number 6 indeed falls within the usual limits for the number of elements that can be stored in working memory, which varies between 4 to 9 elements depending on the authors (Cowan, 2001, 2010; Miller, 1956). Thus, subjects could have solved the violation detection task without compression, purely by storing each 6-items sequence "as is" in working memory. Similarly, 8 -items sequences could have been stored as a mere series of "chunks", which are thought to be the units of encoding in working memory (Cowan, 2010; Cowan, Chen, \& Rouder, 2004; Luck \& Vogel, 1997; Miller, 1956), without any recursive embedding. All in all, an increasingly greater need to rely on compression would explain why the predictive power of LoT complexity increases with sequence length.

Although the definition of working memory chunks as "a collection of elements having strong associations with one another" (Cowan, 2001; Gobet et al., 2001) is too vague to be rigorously tested using the present data, it is easy to imagine that both conceptions can lead to similar predictions (sequences composed of a small number of small chunks also have a short description in our language). Note however that, when considering all tested sequences, LoT complexity outperformed the "number of runs" predictor, for which chunks are defined using consecutive repetitions of the same item. In fact, a crucial feature of our theory lies in going beyond a simple concatenation of chunks and forming 
recursively embedded or nested representations, that is the ability to represent "chunks of chunks" or "repetitions of repetitions". Indeed, the construction of recursively nested structured has been proposed as a core human ability, which sets us apart from other primates (Conway \& Christiansen, 2001; Dehaene et al., 2015; Fitch \& Hauser, 2004; Hauser et al., 2002). Our results support the idea that the inclusion of such feature is essential to explain human behavior when working memory capacity is exceeded and compression is beneficial. The present work provides a foundation for future experiments in non-human primates - we postulate that a simpler language will suffice to account for their sequence coding abilities.

Numerous frameworks for the estimation of pattern complexity have been proposed in the past, such as change complexity (Aksentijevic \& Gibson, 2012), algorithmic complexity (Gauvrit et al., 2016, 2014; Soler-Toscano et al., 2014), subsymmetries (Alexander \& Carey, 1968) or entropy (see also Glanzer \& Clark, 1963; Psotka, 1975; Vitz, 1968, 2019; Vitz \& Todd, 1969). These models are often based on quantitative aspects of information, such as the length, the number of transitions or runs, the probability of those transitions, the number of symmetries, or the number of changes. Although they all show some level of success in predicting behavior, they fail to capture recursive nesting, which as noted above seem to be an essential factor in human cognition (Dehaene et al., 2015; Hauser et al., 2002). Some of these frameworks such as algorithmic complexity employ a more abstract, symbolic approach (in the sense that Turing machines manipulate symbols and use programs to combine them) but merely as an intermediate step, since complexity is only derived from the output frequency of a considerable amount of possible (virtually unknown) machines and programs. In contrast, following others in the domain of concept learning (e.g., Piantadosi et al., 2012, 2016), the approach adopted here starts by assuming that binary sequences are encoded using a specific cognitive system that manipulates abstract, symbolic representations - a language of thought with recursive calls to a limited number of primitive operations. Thus, the present proposal, unlike others, does not merely provide a numerical value for complexity, but also a parse tree and a precise internal format of representation, both of which could possibly be tested in future behavioral or brain-imaging experiments. 
In spite of those successes, a number of questions and potential limitations of this approach remain. First, the construction of our formal language implied methodological choices that could be considered as arbitrary or at least requiring more experimental validation. The primitive instructions included in our formal language were chosen for their alleged simplicity and because they suffice to represent any binary sequence. Other primitives could be tested (e.g. counting and a system of arithmetic; or temporal inversion or "mirroring", see Jiang et al., 2018). Furthermore, modifications of the weights associated with each instruction or their number of repetitions may lead to different estimates of complexity. Finding the correct language for a given population is crucial, especially in the context of the debate on the uniqueness of human sequence processing skills, and specific statistical methodologies need to be developed for this purpose. Bayesian inference, which allows to find the most likely concepts and rules from a grammatically structured hypothesis space containing several candidates (Goodman, Tenenbaum, Feldman, \& Griffiths, 2008), was used by Romano et al. (2018) to show that the primitives included in the language of geometry correspond tightly to a relevant set of rules that best explained behavior (while other primitives do not). Nevertheless, we also found that some of the minimal expressions produced by this language did not fit well with the way participants represent some sequences. The addition of the constraint that the minimal parse tree should respect the chunks or runs of consecutive repetitions, and never split any such chunk, was found to lead to a noticeable improvement in model fit. We speculate that this finding reflects the way participants build their internal representation of sequences: since the space of possible programs is immense, they would restrict the search to only those programs that, at the lowest level, generate the observed consecutive runs in the sequence. The perceptual dominance of the runs could act as a bottleneck, an initial grouping that would then restrict the sequence parsing process (as is sometimes assumed in some complexity estimation models; see e.g. Vitz \& Todd, 1969). A better characterization of this parsing process during sequence learning could help address the current limitations of our language.

Another limitation is that, although we argued that the capacity to represent sequences using hierarchically embedded or nested descriptions is an essential feature of human behavior, about half of the minimal expressions for the sequences that we used included only two hierarchical levels ( a single 
level of embedding) (the average hierarchical depth was 2.5). Only a few sequences such as AABBABABAABBABA explicitly required repetitions of repetitions of repetitions. Although our model correctly predicted their subjective and objective complexity (see Figure 3), and although embedding is an effective compression process, more research is needed to probe whether human participants always consider such deep levels of embedding as beneficial in the processing of short auditory sequences. Increasing the hierarchical depth may imply an additional processing cost, making it useful only in specific situations (e.g. for more demanding learning tasks or with long sequences).

Finally, our approach assumes that the mental compression of sequences does not necessarily occur at the level of the sensory events (i.e. grouping contiguous identical elements) but at the more abstract level of the relationships between events. Besides its success in predicting the psychological complexity of sequences of tones, one argument in favor of such an abstract symbolic representation is that it fitted equally well the complexity of visual binary sequences. However, it could be proposed that the mental encoding of temporal sequence does not involve a modal, domain-general processing mechanisms, but rather two similarly organized modality-specific systems, or even a single modalityspecific cognitive system dedicated to auditory processing; visual sequences would then be converted into an auditory representation prior to compression. Indeed, we observed a lower performance and slower responses in the visual compared to the auditory modality, a difference which has been postulated to reflect a dominance of the auditory system for the encoding of temporal information (Conway \& Christiansen, 2005; Glenberg, Mann, Altman, Forman, \& Procise, 1989; Guttman, Gilroy, \& Blake, 2005). One potential strategy for performing the task of experiment 5 with visual stimuli could have been a subvocal naming of the items, and a maintenance in working memory using the phonological loop (Baddeley, 1992; Baddeley \& Hitch, 1974). Further investigation is required to resolve these points, perhaps by relying on other sensory modalities, by testing transfer across modalities, or by using brainimaging to determine the sensory versus higher-level nature of the brain mechanisms at play. We merely note here that activation of supra-modal prefrontal cortices has been reported during sequence processing (e.g. Huettel et al., 2002; Wang et al., 2019); that the existence of an automatic visual-toauditory conversion in sequence processing has been challenged (McAuley \& Henry, 2010); and that 
the existence of an abstract representation of sequences as proposed here, allowing a transfer of knowledge across modalities, is already supported by some behavioral data (see Yildirim \& Jacobs, 2015).

The violation detection task used in the present study implied the learning of a specific and deterministic sequence in each block, which was repeated multiple times with predictable timings. Our results, however, indicate that the statistical properties of the original sequence were also computed in parallel to the compression process and used for prediction, since, for a given sequence, performance varied according to the level of surprise, i.e. the transition probability of the deviant sound in the context of the current sequence. For equal complexity, we observed a higher accuracy and faster response times for deviants that induced less frequent transitions. The observation that transition probability affects behavior even within a deterministic sequence, as opposed to the stochastic sequences that were used in previous studies of statistical learning (e.g., Huettel et al., 2002; Mars et al., 2008; Garrido et al., 2013; Meyniel et al., 2016; Meyniel and Dehaene, 2017; Maheu et al., 2019), suggests that the learning of transition probabilities between items may occur automatically and in parallel to compression in working memory. This is compatible with the large amount of evidence showing that the brain encodes statistical regularities in sensory inputs in an implicit and unconscious manner (Barascud, Pearce, Griffiths, Friston, \& Chait, 2016; Bendixen, Schröger, \& Winkler, 2009; McDermott, Schemitsch, \& Simoncelli, 2013; Paavilainen, 2013; Saffran et al., 1996). Since the effect of surprise occurred over and above any effect of sequence complexity, it also suggests that this statistical learning system is distinct from the more strategic system based on the learning of the deterministic sequence structure. Again, this is compatible with prior brain imaging results on the local-global paradigm, which indicate that the mismatch negativity (MMN), sensitive to local transition probability, can be dissociated from the P3b response associated with the acquisition of the global sequence (Bekinschtein et al., 2009; Strauss et al., 2015; Wacongne et al., 2011).

When pooling datasets from experiments with different sequence lengths, the linear mixed models with surprise and complexity as predictors fitted the data better than models including one predictor alone, indicating that those two predictors captured distinct variance. However, one may note 
that the size of the surprise effect varied across experiments. Surprise and complexity showed opposite patterns, with a stronger effect of complexity for longer sequences than for short ones and conversely, a strong effect of surprise only with the shortest sequences. Given the evidence that we just cited, showing that transition probabilities are constantly being computed unconsciously, the most likely interpretation is probably that task difficulty increased with sequence length and resulted in longer response times, thus masking the contribution of statistical learning and rendering it more difficult to detect. To evaluate this idea, future work should use event-related potentials such as the MMN, which may provide a more sensitive measure of transition-probability learning.

Finally, we found a complexity effect even when subjects responded to "super-deviants" items, i.e. outlier sounds that could be detected without any knowledge of the sequence because their identity itself was novel. We suggest two putative interpretations of this unexpected effect. First, it could be due to the increased attentional load associated with more complex sequences. Essentially, participants would be placed in a dual-task situation of having to attend to two things are once: the complex sequence and the occasional deviants. In support of this idea, increased attentional load has indeed been found associated to sequence learning impairment in dual-task experiments (see Shanks et al., 2005). A second interpretation, within the predictive coding framework, is that deviance detection, even for extremely salient deviants, is easier for predictable than for unpredictable stimuli. Indeed, Southwell and Chait (2018) found a larger brain response to deviant stimuli within a regular sequence than within a random sequence of tones. The authors propose that it could reflect a difference in the precision or predictability associated with the flow of sensory information. Indeed, in addition to the prediction regarding the content of incoming stimuli (manifested by prediction error signals), recent versions of predictive coding theories also formalize the concept of precision, which corresponds to the reliability of the prediction (Auksztulewicz et al., 2017; Feldman \& Friston, 2010; Heilbron \& Chait, 2018; Rao, 2005). Precision would manifest itself as a gain modulation of the relevant neural units (which is tightly related to attention), with increased precision leading to an increasing sensitivity to the predicted stimuli. This theory can explain the increased and sustained neuronal responses observed in a highly predictable context (Auksztulewicz et al., 2017; Barascud et al., 2016; Southwell et al., 2017; Southwell \& Chait, 
2018). The present complexity effect observed for super-deviants may thus indicate that responses to completely unexpected events were modulated by the degree of predictability of the pattern, which itself depends upon the complexity of the pattern. A precision-weighting mechanism would thus explain why greater complexity leads to slower response times to any kind of violations in our violation detection task. Overall, the distinct contributions of surprise and complexity underline the joint contributions of statistical versus rule-based information in temporal sequence processing.

\section{Conclusion}

Our study provides a first demonstration that responses to violations can be used to uncover the properties of the abstract mental language used by individuals to encode sequential patterns. The present proposal goes beyond previous descriptive theories of sequence complexity, both in the quality of its fit to experimental data, and in the precision of the internal encoding that it proposes. The observed relationship between sequence complexity and performance in the detection of violations is consistent with the idea that the brain acts as a compressor of incoming information that consistently attempts to capture regularities and use them to predict the remainder of the sequence. The present non-verbal passive paradigm paves the way to future neurophysiological recording studies that would probe the similarities and differences between humans and other species (Wang et al., 2015) or test the abilities of preverbal infants (Basirat et al., 2014). A fundamental question for future research is whether the same formal language can explain sequence processing in other primate species, or if such a language is unique to humans (Hauser et al., 2002). 


\section{References}

Abla, D., \& Okanoya, K. (2009). Visual statistical learning of shape sequences: An ERP study. Neuroscience Research, 64(2), 185-190. https://doi.org/10.1016/j.neures.2009.02.013

Akaike, H. (1998). Information Theory and an Extension of the Maximum Likelihood Principle. In E. Parzen, K. Tanabe, \& G. Kitagawa (Eds.), Selected Papers of Hirotugu Akaike (pp. 199-213). https://doi.org/10.1007/978-1-4612-1694-0_15

Aksentijevic, A., \& Gibson, K. (2012). Complexity equals change. Cognitive Systems Research, 15-16, 1-16. https://doi.org/10.1016/j.cogsys.2011.01.002

Alexander, C., \& Carey, S. (1968). Subsymmetries. Perception \& Psychophysics, 4(2), 73-77. https://doi.org/10.3758/BF03209511

Amalric, M., Wang, L., Pica, P., Figueira, S., Sigman, M., \& Dehaene, S. (2017). The language of geometry: Fast comprehension of geometrical primitives and rules in human adults and $\begin{array}{llll}\text { preschoolers. PLOS } & \text { Computational Biology, 13(1), } 1005273 .\end{array}$ https://doi.org/10.1371/journal.pcbi.1005273

Auksztulewicz, R., Barascud, N., Cooray, G., Nobre, A. C., Chait, M., \& Friston, K. (2017). The cumulative effects of predictability on synaptic gain in the auditory processing stream. Journal of Neuroscience, 37(28), 6751-6760.

Baddeley, A. (1992). Working memory. Science, 255(5044), 556-559. https://doi.org/10.1126/science.1736359

Baddeley, A. D., \& Hitch, G. (1974). Working Memory. In G. H. Bower (Ed.), Psychology of Learning and Motivation (Vol. 8, pp. 47-89). https://doi.org/10.1016/S0079-7421(08)60452-1

Barascud, N., Pearce, M. T., Griffiths, T. D., Friston, K. J., \& Chait, M. (2016). Brain responses in humans reveal ideal observer-like sensitivity to complex acoustic patterns. Proceedings of the National Academy of Sciences, 113(5), E616-E625. https://doi.org/10.1073/pnas.1508523113 
Basirat, A., Dehaene, S., \& Dehaene-Lambertz, G. (2014). A hierarchy of cortical responses to sequence violations in three-month-old infants. Cognition, 132(2), 137-150. https://doi.org/10.1016/j.cognition.2014.03.013

Bates, D., Mächler, M., Bolker, B., \& Walker, S. (2015). Fitting Linear Mixed-Effects Models Using lme4. Journal of Statistical Software, 67(1). https://doi.org/10.18637/jss.v067.i01

Bekinschtein, T. A., Dehaene, S., Rohaut, B., Tadel, F., Cohen, L., \& Naccache, L. (2009). Neural signature of the conscious processing of auditory regularities. Proceedings of the National Academy of Sciences, 106(5), 1672-1677. https://doi.org/10.1073/pnas.0809667106

Bendixen, A., Schröger, E., \& Winkler, I. (2009). I heard that coming: Event-related potential evidence for stimulus-driven prediction in the auditory system. The Journal of Neuroscience: The Official Journal of the Society for Neuroscience, 29(26), 8447-8451. https://doi.org/10.1523/JNEUROSCI.1493-09.2009

Brady, T. F., Konkle, T., \& Alvarez, G. A. (2009). Compression in visual working memory: Using statistical regularities to form more efficient memory representations. Journal of Experimental Psychology. General, 138(4), 487-502. https://doi.org/10.1037/a0016797

Brainard, D. H., \& Vision, S. (1997). The psychophysics toolbox. Spatial Vision, 10, 433-436.

Chaitin, G. J. (1969). On the length of programs for computing finite binary sequences: Statistical considerations. Journal of the ACM (JACM), 16(1), 145-159.

Chao, Z. C., Takaura, K., Wang, L., Fujii, N., \& Dehaene, S. (2018). Large-Scale Cortical Networks for Hierarchical Prediction and Prediction Error in the Primate Brain. Neuron, $O(0)$. https://doi.org/10.1016/j.neuron.2018.10.004

Chase, W. G., \& Ericsson, K. A. (1982). Skill and Working Memory. In G. H. Bower (Ed.), Psychology of Learning and Motivation (Vol. 16, pp. 1-58). https://doi.org/10.1016/S0079-7421(08)605460

Chomsky, N. (1957). Syntactic structures (14. printing). The Hague: Mouton.

Conway, C. M., \& Christiansen, M. H. (2001). Sequential learning in non-human primates. Trends in Cognitive Sciences, 5(12), 539-546. https://doi.org/10.1016/S1364-6613(00)01800-3 
Conway, C. M., \& Christiansen, M. H. (2005). Modality-constrained statistical learning of tactile, visual, and auditory sequences. Journal of Experimental Psychology. Learning, Memory, and Cognition, 31(1), 24-39. https://doi.org/10.1037/0278-7393.31.1.24

Cowan, N. (2001). The magical number 4 in short-term memory: A reconsideration of mental storage capacity. Behavioral and Brain Sciences, 24(1), 87-114. https://doi.org/10.1017/S0140525X01003922

Cowan, N. (2010). The Magical Mystery Four: How Is Working Memory Capacity Limited, and Why? Current Directions in Psychological Science, 19(1), 51-57. https://doi.org/10.1177/0963721409359277

Cowan, N., Chen, Z., \& Rouder, J. N. (2004). Constant capacity in an immediate serial-recall task: A logical sequel to Miller (1956). Psychological Science, 15(9), 634-640. https://doi.org/10.1111/j.0956-7976.2004.00732.x

Dehaene, S., Meyniel, F., Wacongne, C., Wang, L., \& Pallier, C. (2015). The Neural Representation of Sequences: From Transition Probabilities to Algebraic Patterns and Linguistic Trees. Neuron, 88(1), 2-19. https://doi.org/10.1016/j.neuron.2015.09.019

Delahaye, J.-P., \& Zenil, H. (2012). Numerical evaluation of algorithmic complexity for short strings: A glance into the innermost structure of randomness. Applied Mathematics and Computation, 219(1), 63-77. https://doi.org/10.1016/j.amc.2011.10.006

Ericsson, K. A., Chase, W. G., \& Faloon, S. (1980). Acquisition of a memory skill. Science (New York, N.Y.), 208(4448), 1181-1182. https://doi.org/10.1126/science.7375930

Faugeras, F., Rohaut, B., Weiss, N., Bekinschtein, T. A., Galanaud, D., Puybasset, L., ... Naccache, L. (2011). Probing consciousness with event-related potentials in the vegetative state. Neurology, 77(3), 264-268. https://doi.org/10.1212/WNL.0b013e3182217ee8

Feldman, H., \& Friston, K. (2010). Attention, Uncertainty, and Free-Energy. Frontiers in Human Neuroscience, 4. https://doi.org/10.3389/fnhum.2010.00215

Feldman, J. (2000). Minimization of Boolean complexity in human concept learning. Nature, 407(6804), 630-633. https://doi.org/10.1038/35036586 
Fitch, W. T. (2014). Toward a computational framework for cognitive biology: Unifying approaches from cognitive neuroscience and comparative cognition. Physics of Life Reviews, 11(3), 329364. https://doi.org/10.1016/j.plrev.2014.04.005

Fitch, W. T., \& Hauser, M. D. (2004). Computational constraints on syntactic processing in a nonhuman primate. Science (New York, N.Y.), 303(5656), 377-380. https://doi.org/10.1126/science.1089401

Fodor, J. A. (1975). The language of thought (Vol. 5). Harvard university press.

Freides, D. (1974). Human information processing and sensory modality: Cross-modal functions, information complexity, memory, and deficit. Psychological Bulletin, 81(5), 284.

Friston, K. (2010). The free-energy principle: A unified brain theory? Nature Reviews Neuroscience, 11(2), 127-138. https://doi.org/10.1038/nrn2787

Fujii, N. (2003). Representation of Action Sequence Boundaries by Macaque Prefrontal Cortical Neurons. Science, 301(5637), 1246-1249. https://doi.org/10.1126/science.1086872

Garrido, M. I., Kilner, J. M., Stephan, K. E., \& Friston, K. J. (2009). The mismatch negativity: A review of underlying mechanisms. Clinical Neurophysiology, 120(3), 453-463. https://doi.org/10.1016/j.clinph.2008.11.029

Garrido, M. I., Sahani, M., \& Dolan, R. J. (2013). Outlier responses reflect sensitivity to statistical structure in the human brain. PLoS Computational Biology, 9(3), e1002999. https://doi.org/10.1371/journal.pcbi.1002999

Gauvrit, N., Singmann, H., Soler-Toscano, F., \& Zenil, H. (2016). Algorithmic complexity for psychology: A user-friendly implementation of the coding theorem method. Behavior Research Methods, 48(1), 314-329. https://doi.org/10.3758/s13428-015-0574-3

Gauvrit, N., Zenil, H., Delahaye, J.-P., \& Soler-Toscano, F. (2014). Algorithmic complexity for short binary strings applied to psychology: A primer. Behavior Research Methods, 46(3), 732-744. https://doi.org/10.3758/s13428-013-0416-0

Gilchrist, A. L., Cowan, N., \& Naveh-Benjamin, M. (2008). Working Memory Capacity for Spoken Sentences Decreases with Adult Aging: Recall of Fewer, but not Smaller Chunks in Older 
$\begin{array}{llll}\text { Adults. } & \text { Memory } & \text { (Hove, } & \text { England), }\end{array}$ https://doi.org/10.1080/09658210802261124

Glanzer, M., \& Clark, W. H. (1963). Accuracy of perceptual recall: An analysis of organization. Journal of Verbal Learning and Verbal Behavior, 1(4), 289-299. https://doi.org/10.1016/S0022$5371(63) 80008-0$

Glenberg, A. M., Mann, S., Altman, L., Forman, T., \& Procise, S. (1989). Modality effects in the coding reproduction of rhythms. Memory \& Cognition, 17(4), 373-383. https://doi.org/10.3758/BF03202611

Gobet, F., Lane, P. C. R., Croker, S., Cheng, P. C.-H., Jones, G., Oliver, I., \& Pine, J. M. (2001). Chunking mechanisms in human learning. Trends in Cognitive Sciences, 5(6), 236-243. https://doi.org/10.1016/S1364-6613(00)01662-4

Goodman, N. D., Tenenbaum, J. B., Feldman, J., \& Griffiths, T. L. (2008). A Rational Analysis of RuleBased Concept Learning. Cognitive Science, 32(1), 108-154. https://doi.org/10.1080/03640210701802071

Goodman, N. D., Ullman, T. D., \& Tenenbaum, J. B. (2011). Learning a theory of causality. Psychological Review, 118(1), 110-119. https://doi.org/10.1037/a0021336

Griffiths, T. L., \& Tenenbaum, J. B. (2003). Probability, algorithmic complexity, and subjective randomness. Proceedings of the Annual Meeting of the Cognitive Science Society, 25.

Grunwald, P. (2004). A tutorial introduction to the minimum description length principle. ArXiv:Math/0406077. Retrieved from http://arxiv.org/abs/math/0406077

Guttman, S. E., Gilroy, L. A., \& Blake, R. (2005). Hearing what the eyes see: Auditory encoding of visual temporal sequences. Psychological Science, 16(3), 228-235. https://doi.org/10.1111/j.0956-7976.2005.00808.x

Hauser, M. D., Chomsky, N., \& Fitch, W. T. (2002). The Faculty of Language: What Is It, Who Has It, and How Did It Evolve? Science, 298(5598), 1569-1579. https://doi.org/10.1126/science.298.5598.1569 
Hauser, M. D., Newport, E. L., \& Aslin, R. N. (2001). Segmentation of the speech stream in a nonhuman primate: Statistical learning in cotton-top tamarins. Cognition, 78(3), B53-B64. https://doi.org/10.1016/S0010-0277(00)00132-3

Hauser, M. D., \& Watumull, J. (2017). The Universal Generative Faculty: The source of our expressive power in language, mathematics, morality, and music. Journal of Neurolinguistics. https://doi.org/10.1016/j.jneuroling.2016.10.005

Heilbron, M., \& Chait, M. (2018). Great Expectations: Is there Evidence for Predictive Coding in $\begin{array}{llll}\text { Auditory } & \text { Cortex? } & \text { Neuroscience, } & 389,\end{array}$ https://doi.org/10.1016/j.neuroscience.2017.07.061

Huettel, S. A., Mack, P. B., \& McCarthy, G. (2002). Perceiving patterns in random series: Dynamic processing of sequence in prefrontal cortex. Nature Neuroscience, 5(5), 485-490. https://doi.org/10.1038/nn841

Jiang, X., Long, T., Cao, W., Li, J., Dehaene, S., \& Wang, L. (2018). Production of Supra-regular Spatial Sequences by Macaque Monkeys. Current Biology: CB, 28(12), 1851-1859.e4. https://doi.org/10.1016/j.cub.2018.04.047

Kenward, M. G., \& Roger, J. H. (1997). Small sample inference for fixed effects from restricted maximum likelihood. Biometrics, 53(3), 983-997.

Kirkham, N. Z., Slemmer, J. A., \& Johnson, S. P. (2002). Visual statistical learning in infancy: Evidence for a domain general learning mechanism. Cognition, 83(2), B35-B42. https://doi.org/10.1016/S0010-0277(02)00004-5

Kleiner, M., Brainard, D., Pelli, D., Ingling, A., Murray, R., \& Broussard, C. (2007). What's new in Psychtoolbox-3. Perception, 36(14), 1.

Kolmogorov, A. N. (1968). Three approaches to the quantitative definition of information. International Journal of Computer Mathematics, 2(1-4), 157-168.

Kuznetsova, A., Brockhoff, P. B., \& Christensen, R. H. (2017). lmerTest package: Tests in linear mixed effects models. Journal of Statistical Software, 82(13), 1-26. 
Lashley, K. S. (1951). The problem of serial order in behavior. In L. A. Jeffress \& L. A. (Ed) Jeffress (Eds.), Cerebral mechanisms in behavior; the Hixon Symposium. (pp. 112-146). Oxford, England: Wiley. (1952-04498-003).

Luck, S. J., \& Vogel, E. K. (1997). The capacity of visual working memory for features and conjunctions. Nature, 390(6657), 279-281. https://doi.org/10.1038/36846

Maheu, M., Dehaene, S., \& Meyniel, F. (2019). Brain signatures of a multiscale process of sequence learning in humans. ELife, 8. https://doi.org/10.7554/eLife.41541

Marcus, G. F., Vijayan, S., Bandi Rao, S., \& Vishton, P. M. (1999). Rule learning by seven-month-old infants. Science, 283(5398), 77-80. (9872745).

Mars, R. B., Debener, S., Gladwin, T. E., Harrison, L. M., Haggard, P., Rothwell, J. C., \& Bestmann, S. (2008). Trial-by-Trial Fluctuations in the Event-Related Electroencephalogram Reflect Dynamic Changes in the Degree of Surprise. Journal of Neuroscience, 28(47), 12539-12545. https://doi.org/10.1523/JNEUROSCI.2925-08.2008

McAuley, J. D., \& Henry, M. J. (2010). Modality effects in rhythm processing: Auditory encoding of visual rhythms is neither obligatory nor automatic. Attention, Perception, \& Psychophysics, 72(5), 1377-1389. https://doi.org/10.3758/APP.72.5.1377

McDermott, J. H., Schemitsch, M., \& Simoncelli, E. P. (2013). Summary statistics in auditory perception. Nature Neuroscience, 16(4), 493-498. https://doi.org/10.1038/nn.3347

Meyer, T., \& Olson, C. R. (2011). Statistical learning of visual transitions in monkey inferotemporal cortex. Proceedings of the National Academy of Sciences, 108(48), 19401-19406. https://doi.org/10.1073/pnas.1112895108

Meyniel, F., \& Dehaene, S. (2017). Brain networks for confidence weighting and hierarchical inference during probabilistic learning. Proceedings of the National Academy of Sciences, 114(19), E3859-E3868. https://doi.org/10.1073/pnas.1615773114

Meyniel, F., Maheu, M., \& Dehaene, S. (2016). Human Inferences about Sequences: A Minimal Transition Probability Model. PLOS Computational Biology, 12(12), e1005260. https://doi.org/10.1371/journal.pcbi.1005260 
Miller, G. A. (1956). The magical number seven, plus or minus two: Some limits on our capacity for processing information. Psychological Review, 63(2), 81-97. https://doi.org/10.1037/h0043158

Näätänen, R. (2003). Mismatch negativity: Clinical research and possible applications. International Journal of Psychophysiology, 48(2), 179-188. https://doi.org/10.1016/S0167-8760(03)00053-9

Paavilainen, P. (2013). The mismatch-negativity (MMN) component of the auditory event-related potential to violations of abstract regularities: A review. International Journal of Psychophysiology: Official Journal of the International Organization of Psychophysiology, 88(2), 109-123. https://doi.org/10.1016/j.jpsycho.2013.03.015

Patel, A. D., Iversen, J. R., Chen, Y., \& Repp, B. H. (2005). The influence of metricality and modality on synchronization with a beat. Experimental Brain Research, 163(2), 226-238. https://doi.org/10.1007/s00221-004-2159-8

Piantadosi, S. T., \& Jacobs, R. A. (2016). Four Problems Solved by the Probabilistic Language of Thought. Current Directions in Psychological Science, 25(1), 54-59. https://doi.org/10.1177/0963721415609581

Piantadosi, S. T., Tenenbaum, J. B., \& Goodman, N. D. (2012). Bootstrapping in a language of thought: A formal model of numerical concept learning. Cognition, 123(2), 199-217. https://doi.org/10.1016/j.cognition.2011.11.005

Piantadosi, S. T., Tenenbaum, J. B., \& Goodman, N. D. (2016). The logical primitives of thought: Empirical foundations for compositional cognitive models. Psychological Review, 123(4), 392424. https://doi.org/10.1037/a0039980

Psotka, J. (1975). Simplicity, symmetry, and syntely: Stimulus measures of binary pattern structure. Memory \& Cognition, 3(4), 434-444. https://doi.org/10.3758/BF03212938

R Core Team. (2017). R: A language and environment for statistical computing. Retrieved from https://www.R-project.org/

Rao, R. P. N. (2005). Bayesian inference and attentional modulation in the visual cortex. NeuroReport, 16(16), 1843. https://doi.org/10.1097/01.wnr.0000183900.92901.fc

Restle, F. (1970). Theory of serial pattern learning: Structural trees. Psychological Review, 77(6), 481495. https://doi.org/10.1037/h0029964 
Restle, F. (1973). Serial pattern learning: Higher order transitions. Journal of Experimental Psychology, 99(1), 61-69. https://doi.org/10.1037/h0034751

Restle, F., \& Brown, E. R. (1970). Serial Pattern Learning. Journal of Experimental Psychology, 83(1, Pt.1), 120-125. https://doi.org/10.1037/h0028530

Romano, S., Salles, A., Amalric, M., Dehaene, S., Sigman, M., \& Figueira, S. (2018). Bayesian validation of grammar productions for the language of thought. PloS One, 13(7), e0200420. https://doi.org/10.1371/journal.pone.0200420

Romano, S., Sigman, M., \& Figueira, S. (2013). $\$ \mathrm{LT}^{\wedge} 2 \mathrm{C}^{\wedge} 2 \$$ : A language of thought with Turingcomputable Kolmogorov complexity. Papers in Physics, 5, 050001-050001. https://doi.org/10.4279/pip.050001

Romberg, A. R., \& Saffran, J. R. (2010). Statistical learning and language acquisition. Wiley Interdisciplinary Reviews: Cognitive Science, 1(6), 906-914. https://doi.org/10.1002/wcs.78

Saffran, J. R., Aslin, R. N., \& Newport, E. L. (1996). Statistical Learning by 8-Month-Old Infants. Science, 274(5294), 1926-1928. https://doi.org/10.1126/science.274.5294.1926

Shanks, D. R., Rowland, L. A., \& Ranger, M. S. (2005). Attentional load and implicit sequence learning. Psychological Research, 69(5), 369-382. https://doi.org/10.1007/s00426-004-0211-8

Shannon, C. E. (1948). A mathematical theory of communication. Bell System Technical Journal, 27(3), $379-423$.

Soler-Toscano, F., Zenil, H., Delahaye, J.-P., \& Gauvrit, N. (2014). Calculating Kolmogorov Complexity from the Output Frequency Distributions of Small Turing Machines. PLoS ONE, 9(5), e96223. https://doi.org/10.1371/journal.pone.0096223

Southwell, R., Baumann, A., Gal, C., Barascud, N., Friston, K., \& Chait, M. (2017). Is predictability salient? A study of attentional capture by auditory patterns. Philosophical Transactions of the Royal Society of London. Series B, Biological Sciences, 372(1714). https://doi.org/10.1098/rstb.2016.0105

Southwell, Rosy, \& Chait, M. (2018). Enhanced deviant responses in patterned relative to random sound sequences. Cortex; a Journal Devoted to the Study of the Nervous System and Behavior, 109, 92-103. https://doi.org/10.1016/j.cortex.2018.08.032 
Squires, N. K., Squires, K. C., \& Hillyard, S. A. (1975). Two varieties of long-latency positive waves evoked by unpredictable auditory stimuli in man. Electroencephalography and Clinical Neurophysiology, 38(4), 387-401. https://doi.org/10.1016/0013-4694(75)90263-1

Strange, B. A., Duggins, A., Penny, W., Dolan, R. J., \& Friston, K. J. (2005). Information theory, novelty and hippocampal responses: Unpredicted or unpredictable? Neural Networks, 18(3), 225-230. https://doi.org/10.1016/j.neunet.2004.12.004

Strauss, M., Sitt, J. D., King, J.-R., Elbaz, M., Azizi, L., Buiatti, M., ... Dehaene, S. (2015). Disruption of hierarchical predictive coding during sleep. Proceedings of the National Academy of Sciences, 112(11), E1353-E1362. https://doi.org/10.1073/pnas.1501026112

Toussaint, G. T., \& Beltran, J. F. (2013). Subsymmetries predict auditory and visual pattern complexity. Perception, 42(10), 1095-1100. https://doi.org/10.1068/p7614

Uhrig, L., Dehaene, S., \& Jarraya, B. (2014). A Hierarchy of Responses to Auditory Regularities in the Macaque Brain. Journal of Neuroscience, 34(4), 1127-1132. https://doi.org/10.1523/JNEUROSCI.3165-13.2014

Vandierendonck, A. (2017). A comparison of methods to combine speed and accuracy measures of performance: A rejoinder on the binning procedure. Behavior Research Methods, 49(2), 653673. https://doi.org/10.3758/s13428-016-0721-5

Vandierendonck, A. (2018). Further Tests of the Utility of Integrated Speed-Accuracy Measures in Task Switching. Journal of Cognition, 1(1). https://doi.org/10.5334/joc.6

Vitz, P. C. (1968). Information, run structure and binary pattern complexity. Perception \& Psychophysics, 3(4), 275-280. https://doi.org/10.3758/BF03212743

Vitz, P. C. (2019). A hierarchical model of binary pattern learning. Learning and Motivation, 65, 5259. https://doi.org/10.1016/j.lmot.2019.01.002

Vitz, P. C., \& Todd, T. C. (1969). A coded element model of the perceptual processing of sequential stimuli. Psychological Review, 76(5), 433-449. https://doi.org/10.1037/h0028113

Wacongne, C., Labyt, E., Wassenhove, V. van, Bekinschtein, T., Naccache, L., \& Dehaene, S. (2011). Evidence for a hierarchy of predictions and prediction errors in human cortex. Proceedings of 
the National Academy of Sciences, 108(51), 20754-20759. https://doi.org/10.1073/pnas.1117807108

Wagenmakers, E.-J., \& Farrell, S. (2004). AIC model selection using Akaike weights. Psychonomic Bulletin \& Review, 11(1), 192-196. https://doi.org/10.3758/BF03206482

Wang, L., Amalric, M., Fang, W., Jiang, X., Pallier, C., Figueira, S., ... Dehaene, S. (2019). Representation of spatial sequences using nested rules in human prefrontal cortex. NeuroImage, 186, 245-255. https://doi.org/10.1016/j.neuroimage.2018.10.061

Wang, L., Uhrig, L., Jarraya, B., \& Dehaene, S. (2015). Representation of Numerical and Sequential Patterns in Macaque and Human Brains. Current Biology, 25(15), 1966-1974. https://doi.org/10.1016/j.cub.2015.06.035

Wilson, B., Marslen-Wilson, W. D., \& Petkov, C. I. (2017). Conserved Sequence Processing in Primate $\begin{array}{lllll}\text { Frontal } & \text { Cortex. Trends in } & \text { Neurosciences, }\end{array}$ https://doi.org/10.1016/j.tins.2016.11.004

Wilson, B., Slater, H., Kikuchi, Y., Milne, A. E., Marslen-Wilson, W. D., Smith, K., \& Petkov, C. I. (2013). Auditory Artificial Grammar Learning in Macaque and Marmoset Monkeys. Journal of Neuroscience, 33(48), 18825-18835. https://doi.org/10.1523/JNEUROSCI.2414-13.2013

Yildirim, I., \& Jacobs, R. A. (2015). Learning multisensory representations for auditory-visual transfer of sequence category knowledge: A probabilistic language of thought approach. Psychonomic Bulletin \& Review, 22(3), 673-686. https://doi.org/10.3758/s13423-014-0734-y 